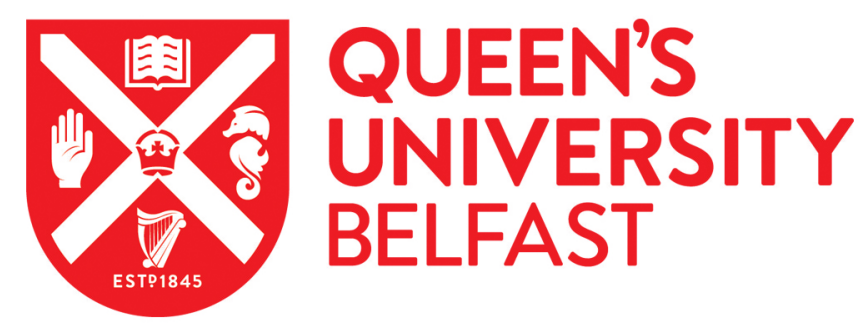

\title{
Ab initio study of the structure, isotope effects, and vibrational properties in KDP crystals
}

Menchón, R., Colizzi, G., Johnston, C., Torresi, F., Lasave, J., Koval, S., Kohanoff, J., \& Migoni, R. (2018). Ab initio study of the structure, isotope effects, and vibrational properties in KDP crystals. Physical Review B, 98(10), [104108]. https://doi.org/10.1103/PhysRevB.98.104108

Published in:

Physical Review B

Document Version:

Peer reviewed version

Queen's University Belfast - Research Portal:

Link to publication record in Queen's University Belfast Research Portal

Publisher rights

(02018 American Physical Society. This work is made available online in accordance with the publisher's policies. Please refer to any applicable terms of use of the publisher.

\section{General rights}

Copyright for the publications made accessible via the Queen's University Belfast Research Portal is retained by the author(s) and / or other copyright owners and it is a condition of accessing these publications that users recognise and abide by the legal requirements associated with these rights.

Take down policy

The Research Portal is Queen's institutional repository that provides access to Queen's research output. Every effort has been made to ensure that content in the Research Portal does not infringe any person's rights, or applicable UK laws. If you discover content in the Research Portal that you believe breaches copyright or violates any law, please contact openaccess@qub.ac.uk. 


\title{
Ab initio study of the structure, isotope effects and vibrational properties in KDP crystals
}

\author{
R. Menchón, ${ }^{1}$ G. Colizzi, ${ }^{2}$ C. Johnston,${ }^{2}$ F. Torresi,${ }^{1}$ J. Lasave, ${ }^{1}$ S. Koval,,${ }^{1}$ J. Kohanoff, ${ }^{2}$ and R. Migoni ${ }^{1}$ \\ ${ }^{1}$ Instituto de Física Rosario, Universidad Nacional de Rosario and CONICET, \\ 27 de Febrero 210 Bis, 2000 Rosario, Argentina \\ ${ }^{2}$ Atomistic Simulation Centre, Queen's University Belfast, Northern Ireland, UK
}

(Dated: August 10, 2018)

\begin{abstract}
The lattice dynamics of potassium dihydrogen phosphate (KDP) and its deuterated analog DKDP was studied via first-principles DFT calculations. A thorough assessment of the quality of a wide range of functionals supplemented with the approximate inclusion of quantum nuclear effects indicated that the non-local van der Waals functional vdW-DF [M. Dion et al, Phys. Rev. Lett. 92, 246401 (2004); J. Klimeš et al, Phys. Rev. B 83, 195131 (2011)] produces the best agreement with structural data for both compounds. This enabled the calculation of full phonon dispersions in the ferroelectric phase, and hence the phonon density of states and specific heat, in excellent agreement with experimental data. Phonon bands and especially modes at the $\Gamma$-point of the Brillouin zone were classified according to their vibrational pattern. This allowed for the assignment of stretching and bending modes of the hydrogen bonds. Internal modes involving the phosphate units were identified at lower frequencies, while the lowest-lying modes were those involving the $\mathrm{K}^{+}$ ion. These assignments were used to interpret infrared and Raman spectra along the $c$-axis, and in the perpendicular plane. Phonon modes calculated at the $\Gamma$-point showed two types of instabilities. One was a normal mode polarized along the $c$-axis of the crystal, while the other corresponded to a two-fold degenerate mode polarized in the perpendicular plane. The former gives rise to a spontaneous polarization in the ferroelectric phase at low temperatures by coupling to an optical $\mathrm{K}^{+}-\mathrm{PO}_{4}^{-}$ stretching mode, consistently with a significant off-diagonal Born effective charge on the hydrogen atoms. A mode describing the opposite rotation of neighboring $\mathrm{PO}_{4}$ tetrahedra was also found to couple strongly to the ferroelectric mode, as this modulates the $\mathrm{O}-\mathrm{O}$ distance, which determines the barrier for proton transfer. The present study suggests that a minimal model to describe isotope effects in KDP should involve at least three fully-coupled vibrational modes.
\end{abstract}

PACS numbers: 77.84.Fa, 71.15.Nc, 77.80.-e, 63.20.Dj

\section{INTRODUCTION}

Potassium dihydrogen phosphate $\left(\mathrm{KH}_{2} \mathrm{PO}_{4}\right.$, or KDP $)$ stands out as the prototype member of the large family of H-bonded ferroelectric compounds, which was extensively studied since the discovery of KDP's ferroelectric properties in $1935 .^{1-4}$ Its importance lies in the numerous technological applications, e.g. as a key component in optoelectronic devices, as well as from a fundamental point of view for its physical properties. The most striking manifestation of its phenomenogy is, without doubt, the huge isotope effect in its ferroelectric-paraelectric (FE$\mathrm{PE})$ transition temperature $\mathrm{T}_{c}$, which changes from $\approx$ $122 \mathrm{~K}$ to $\approx 229 \mathrm{~K}$ with deuteration. This isotope effect is a common feature in the whole family of $\mathrm{H}$-bonded $\mathrm{FE}$ compounds.

In KDP, phosphate groups are linked through nearly planar H-bonds forming a complex three-dimensional network (see Fig. 1). In the high-temperature PE phase, protons are distributed with equal probability along two symmetrical positions separated a distance $\delta=d_{O O}-2 d_{O H}$ along the H-bond, with $d_{O O}$ and $d_{O H}$ the $\mathrm{O}-\mathrm{O}$ and $\mathrm{O}-\mathrm{H}$ distances, respectively. ${ }^{5,6}$ Below $\mathrm{T}_{c}$, the symmetry is broken and protons collectively drop into one of the two symmetric sites, thus leading to the ordered FE phase in KDP. In this phase, the ice rules are fulfilled, i.e. each $\mathrm{PO}_{4}$ group has two chemically bonded protons and forms two hydrogen bonds with neighboring phosphates. The FE instability has its origin in the $\mathrm{H}$ off-centering along the H-bonds, which also leads to the development of a spontaneous polarization $P_{s}$ along the z-direction. $^{3}$

The first explanation of the isotope effect in KDP was provided by the tunneling model proposed in the early sixties by Blinc. ${ }^{7}$ In this model, interacting protons/deuterons tunnel individually through the barrier of a double-well potential along the H-bond. For protons, the tunneling particle is delocalized in the H-bond thus favoring the onset of disorder at a lower $T_{c}$ than that corresponding to the deuterated case. This mechanism and subsequent related modifications have been considered at the root of the isotope effect for several decades. ${ }^{8-10}$ Since the late eighties, however, extensive neutron diffraction experiments under pressure have revealed the relevance of geometrical effects in the transition temperature, due to deuteration..$^{5,6,11-13}$ The controversy was further fuelled by the observation, in 2002, of tunneling in the PE phase of KDP by neutron Compton scattering experiments. ${ }^{14}$ With the aid of first principles calculations, we have shown that tunneling and geometric effects are complementary aspects of the same phenomenon. 3,15 That is, they self-consistently amplify each other, leading to the huge isotope effect observed.

The dynamical characteristics of the phase transi- 
tion in KDP were also largely debated in the past, as to whether it is a displacive or an order-disorder FE transition. ${ }^{9,16}$ Originally, the coupled proton-phonon model, which displays essentially a displacive-like transition, was proposed to explain some experimental facts. This model consists of proton collective modes, which arise as a consequence of the tunneling motion along the H-bonds in the basal plane. ${ }^{17}$ The interaction of these modes with an optical lattice phonon of $\mathrm{B}_{2}$ symmetry polarized along the $z$-axis (stretching of the $\mathrm{K}^{+}-\mathrm{PO}_{4}{ }^{3-}$ complex) was invoked to explain the emergence of polarization in the $z$-direction in the FE phase. ${ }^{8,18}$ One of the two phonons arising from these interactions is precisely the FE soft-mode $\omega_{-}$which softens as the critical temperature is reached. However, the interacting-phonons scenario seems to be even more complex according to infrared measurements. ${ }^{18}$ It is observed that an overdamped internal mode $\nu_{4}$ related to a quadrupole distortion of the phosphates shifts down in frequency together with the soft-mode $\omega_{-}$and the lattice externalphonon $\mathrm{B}_{2}$ as $\mathrm{T}$ increases approaching $\mathrm{T}_{c}$. The coupled excitations, which should be described by Fano-type interactions, evolve into a quasi-continuum with a highly overdamped central component near $\mathrm{T}_{c}$. These findings are consistent with a displacive-type transition which was also suggested by very recent magic angle spinning NMR experiments. ${ }^{19}$ On the other hand, dielectric dispersion ${ }^{20}$ and Brillouin scattering ${ }^{21}$ experiments have revealed that the dielectric anomaly at $\mathrm{T}_{c}$ is not due to a softening of $\omega_{-}$but to a critical slowing down of a relaxational polarization fluctuation. ${ }^{16}$ Moreover, Raman scattering results show some forbidden peaks in the PE-phase spectra which correspond to internal modes of $\mathrm{PO}_{4}$ in the geometry assumed by the tetrahedra below $\mathrm{T}_{c} \cdot{ }^{22}$ The latter results are more compatible with an order-disorder transition of the $\mathrm{H}_{2} \mathrm{PO}_{4}$ dipoles than with a displacive-like behavior promoted by phonon softening. , $^{9,23}$

Raman $^{22,24-28}$ and infrared ${ }^{18,29}$ measurements have provided substantial information about the lattice dynamics of KDP decades ago. From the theoretical side, however, attemps to model the system in order to calculate phonons were scarce in the past. ${ }^{30}$ We have recently developed a shell model for KDP which was adjusted to ab initio data. ${ }^{31}$ The model reproduces reasonably well some structural, energetic and vibrational properties of the material. The emphasis in Ref. ${ }^{31}$ was specifically placed in developing the shell model but not in describing comprehensively the lattice dynamics of KDP at the first principles level. Calculations of some of the zonecenter phonons for KDP at the same level have been also performed recently in Ref. ${ }^{32}$. However, the limited qualitative analysis of these phonons have served only to the purpose of describing some anharmonic features related to the soft-mode. In spite of the importance that phonons have in the proper understanding of the microscopic mechanism of the FE transition in these compounds, to our best knowledge, no comprehensive ab initio calculations of the vibrational properties of KDP were performed to date. An ab initio study has the advantage of providing a confident parameter-free analysis of the frequencies and eigenvectors of the phonons. This advantage becomes clear when considering very recent model $^{33,34}$ and first-principles ${ }^{35}$ calculations of the lattice dynamics of $\mathrm{CsH}_{2} \mathrm{PO}_{4}$, a $\mathrm{H}$-bonded $\mathrm{FE}$ compound that belongs to the KDP family. In Ref. ${ }^{35}$ is concluded that dispersion corrections to semi-local density functionals are necessary to correctly describe the phonons of the system.

In this work, we investigate the vibrational and structural properties of KDP by using density functional theory (DFT) electronic structure calculations. The calculations performed here aim at characterizing: (1) the structure of the PE and FE phases, (2) energy barriers for proton transfer, (3) phonons and normal modes in the Brillouin zone center, (4) full phonon dispersion curves, and 5) phonon density of states and specific heat. The results will be compared to the available experimental data. The phonon calculations in the PE phase exhibit a number of unstable modes with imaginary frequencies. Among them, we identify a mode that is responsible for the ferroelectric distortion, and also a number of other vibrational modes that couple with it at large amplitudes, beyond the limit of validity of the harmonic approximation. These couplings are important because they stabilize the crystal above the transition temperature.

A delicate issue concerns the choice of the exchangecorrelation functional in the calculations because the $\mathrm{H}-$ bond geometry and the global proton-transfer energy barriers depend substantially on it. ${ }^{36-38}$ Thus, as a first step we analyze the performance of different ab initio methods and approximations in describing the geometry and energy barriers associated to the $\mathrm{H}$-bonds in the system. In doing this, we also include phenomenologically the correction to the first principles $\mathrm{H}$-bond classical geometry due to quantum nuclear effects. ${ }^{39}$ This is done by performing Path Integral Monte Carlo (PIMC) simulations for a simple model adjusted to ab initio data. After choosing the best first-principles method for describing the H-bond properties, we then performed the ab initio calculations to determine the vibrational properties of the system.

The paper is organized as follows: in Sec. II we describe the ab initio (AI) methods and computational details. We present the structural and energy results in Section III, which is divided in two subsections as follows. The validation of the ab initio methods and the analysis of the structural and energy results are described in Subsection III.A. A simple model adjusted to AI data is presented in Subsection III.B which is used to determine the nuclear quantum corrections to the H-bond geometry. Section IV is devoted to the analysis of the ab initio results obtained for the phonons and related vibrational properties of the system and is divided in four Subsections. A symmetry analysis of the modes at the Brillouin zone center is presented in Subsection IV.A. The results for the zone-center phonons and its comparison 
with available experimental data are reported in Subsection IV.B. We also describe in this subsection the phonon dispersion curves calculated along the main symmetry directions of the Brillouin zone and the corresponding total and partial phonon density of states. Results for the specific heat and effective Debye temperature, derived from the total density of states, are analyzed and compared to experimental data in Subsection IV.C. Subsection IV.D is devoted to the analysis of the interactions between the unstable FE mode and other phonons which are relevant to the FE phase transition. This part is an extension of the preliminary analysis of the coupling effects presented in Ref. ${ }^{32}$, which was of limited extent. Finally, in section $\mathrm{V}$ we discuss the results and elaborate our conclusions.

\section{AB INITIO METHODS AND CALCULATION DETAILS}

Two different ab initio DFT codes, both based on a plane-wave expansion of the one-electron orbitals, were used to calculate structural properties and phonons at the Brillouin zone center in the $\mathrm{FE}$ and $\mathrm{PE}$ phases. These are the QUANTUM ESPRESSO (QE) program ${ }^{40}$ based on a plane-wave pseudopotential approach and the VASP code ${ }^{41,42}$ which enabled us to use projector augmented wave (PAW) all-electron potentials. Some additional calculations were carried out with the $\mathrm{CP} 2 \mathrm{~K}$ package, ${ }^{43}$ which uses the gaussian and plane wave (GPW) method. ${ }^{44-46}$ The phonon dispersion curves were evaluated using the Finite Difference (FD) method as implemented in the PHONOPY package, ${ }^{47}$ and using the VASP program to compute forces on the atoms via the Hellmann-Feynman theorem. The FD method is based on the ab initio force-constant approach in which the force constant matrix is derived from the ab initio calculation of forces on the atoms induced by the displacements of other atoms in a supercell. ${ }^{48}$ Once the force constants are obtained, the dynamical matrix can be determined at different q-points along the main symmetry directions of the Brillouin zone.

In the calculations performed with QE, we used nonlocal norm-conserving pseudopotentials of the TroullierMartins type ${ }^{49}$ to integrate out the core electrons plus frozen ionic cores. Non-linear core corrections were aditionally included for a proper description of the potassium ions. The energy cutoff in the plane-wave expansion was set to $100 \mathrm{Ry}(1360 \mathrm{eV})$, which yielded total-energy results of sufficient accuracy.

In the VASP calculations, the plane wave basis was expanded to an energy cutoff of $750 \mathrm{eV}$, except for the case of the hybrid exchange-correlation (XC) functional PBE0, in which the energy cutoff was set to $450 \mathrm{eV}$. All the atomic relaxations were carried out until the forces on every mobile atom were smaller than $5 \mathrm{meV} / \AA$.

Calculations with both VASP and QE were carried out using an automatic Monkhorst-Pack $5 \times 5 \times 5$ grid sampling of the electronic Brillouin zone, except for the cases of XC functionals PBE0 and HSE06 in which a $4 \times 4 \times 4$ grid was used instead. In all cases, the mentioned grids proved sufficient to achieve converged results.

In the $\mathrm{CP} 2 \mathrm{~K}$ calculations, the core electrons were replaced by Goedecker-Teter-Hutter-type relativistic, norm-conserving pseudopotentials. ${ }^{50,51}$ Dunning type correlation-consistent triple-zeta gaussian basis sets were used to expand the one-electron orbitals. The Auxiliary Density Matrix Method ${ }^{52,53}$ (ADMM) was used for the Hartree-Fock exchange when performing calculations with hybrid functionals. However, for the MP2 calculations, full Hartree-Fock was used. The resolution-of-identity method was exploited for the MP2 calculations. ${ }^{54-56}$ For the plane wave basis, the energy cutoff was set to $1200 \mathrm{Ry}$. In all of the CP2K calculations, supercells were employed and the Brillouin zone was sampled only at the $\Gamma$-point. For the condensed phase MP2 calculations, the computational expense of the method limited the system to a $2 \times 2 \times 1$ supercell. Calculations at the GGA and hybrid levels of theory used the same supercell for the sake of comparison. The atomic positions were optimised at each level of theory, including MP2.

The various exchange-correlation functionals for the methods used in this work are presented in the next section, where we perform a complete analysis of their performance.

In the calculation of the phonon dispersion curves along the main symmetry directions of the Brillouin zone with the PHONOPY package, the atomic forces were determined using the VASP program with an energy cutoff of $450 \mathrm{eV}$ and a $4 \times 4 \times 4$ grid for the Brillouin-zone sampling. The atomic distortions were performed in a $2 \times 2 \times 2$ supercell with 128 atoms. Here, a tighter tolerance in the forces of $0.5 \mathrm{meV} / \AA$ was imposed on every mobile atom, to achieve convergence in the phonon-dispersion results.

The PE phase of KDP is tetragonal with shorter lattice constant along the tetragonal axis. The space group is $\mathrm{I} \overline{4} 2 \mathrm{~d}$ or $\mathrm{D}_{2 d}^{12}$. In the FE phase the crystal is $0.8 \%$ shear distorted along the [110] direction and becomes orthorhombic with space group Fdd2 or $\mathrm{C}_{2 v}^{19}$ (See Fig. 1).

The polarization develops along the tetragonal axis of the PE phase (z-direction). The primitive cell in both phases contains 16 atoms (two formula units). We used the experimental lattice constants which were fixed during all the simulations. For the initial coordinates of the $\mathrm{P}, \mathrm{K}$, and $\mathrm{O}$ atoms, we used the corresponding Wyckoff positions of the space groups for the PE and FE phases of KDP. ${ }^{57}$ In the PE phase, hydrogens occupy with equal probabilities two symmetric positions along the O-H $\cdots \mathrm{O}$ bond and hence the averaged proton position is $\langle x\rangle=0$. Thus, in this case we performed structural optimizations with the $\mathrm{H}$ atoms exactly at the middle of the $\mathrm{O}-\mathrm{H}-\mathrm{O}$ bonds, which remained in their positions after relaxations due to symmetry reasons. ${ }^{3,38}$ In order to obtain the FE phase, the $\mathrm{H}$ atoms were initially slightly displaced from the middle of the O-H-O bonds towards one of the oxygens following the pattern of the FE soft-mode, and then 


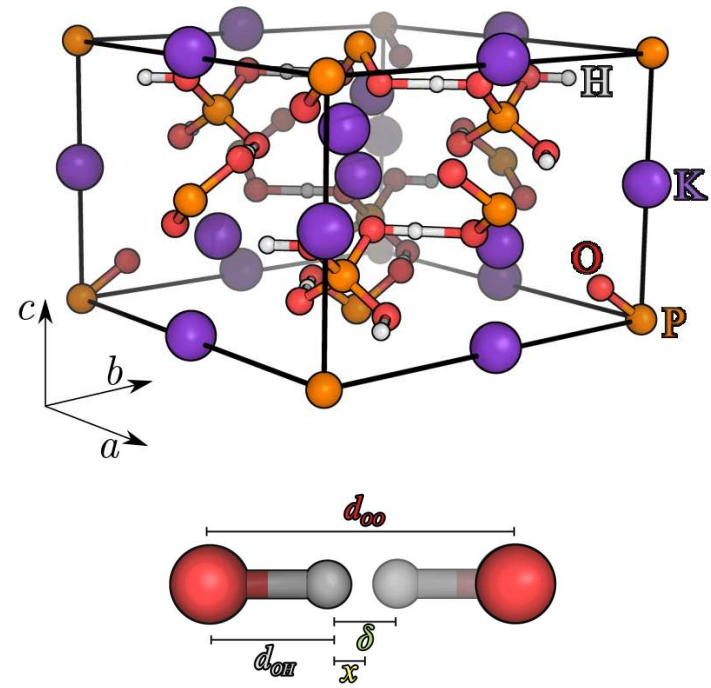

Figure 1: Top panel: schematic view of the internal structure of KDP in the FE phase. Bottom panel: schematic representation of the H-bond with its internal parameters.

a full atomic relaxation was performed. ${ }^{3}$

\section{STRUCTURE AND ENERGY BARRIERS}

\section{A. Structural analysis and validation of XC functionals}

It is well-known that, in H-bonded complexes, the O$O$ distance, $d_{O O}$, and the parameter $\delta$ defined above depend crucially on the XC functional used. In particular, the geometry depends substantially on whether van der Waals (vdW) dispersion corrections are included or not. These geometric modifications are accompanied by variations in the energy barriers associated to proton transfer along the H-bonds (global energy barriers), which in turn influence $\mathrm{T}_{c}{ }^{36-38}$

To validate the ab initio schemes with different $\mathrm{XC}$ functionals, structural optimizations were performed for KDP using the QE and VASP codes, as described in Section II. The performance of several non-local vdW functionals: vdW-DF ${ }^{58-60}$, vdW-DF2 ${ }^{61}$, optB88$\mathrm{vdW}^{59,62}$ and optPBE-vdW $\mathrm{W}^{59,62}$ was analyzed, as well as that of simplified vdW approaches like the DFT-D2 scheme proposed by Grimme ${ }^{63}$ and that of Tkatchenko and Scheffler $[\mathrm{vdW}(\mathrm{TS})]^{64}$. We also performed calculations with the semi-local GGA Perdew-Burke-Ernzerhof (PBE) functional, ${ }^{65}$ which does not account for $\mathrm{vdW}$ long-range interactions, and analyzed the performance of the hybrid functionals $\mathrm{PBE} 0^{66}$, HSE06 ${ }^{67,68}$, and M06$2 \mathrm{X} .{ }^{69}$ In order to compare with the experimental results for the H-bond geometry, the effect of nuclear quantum corrections (NQC) on the structural results (Ubbelohde effect) is approximately included in some of the calcula- tions (see results in parenthesis in Table I). These corrections also include approximately the effect of thermal fluctuations in the H-bond geometry. The details of the nuclear quantum calculations are given in Section III B.

The results of proton-transfer energy barriers and $\mathrm{H}-$ bond geometries calculated with different AI schemes are shown and compared to the experiment in Table I. The lowest energy barriers and shortest $d_{O O}$ and $\delta$ variables correspond to the PBE calculations. In this case, NQC applied to the H-bond geometry in KDP yield too small values for both $d_{O O}$ and $\delta$, when compared to the corresponding experimental data. The empirical dispersion correction DFT-D2 gives values for the barrier and the distances comparable to the PBE case. The calculated energy barriers and $d_{O O}$ and $\delta$ parameters are more significant in the case of the non-local vdW method optPBE-vdW. However, the introduction of NQC yields a contracted geometry compared to the experiment, with a remarkable disagreement for the parameter $\delta$. Hybrid functionals (PBE0, HSE06, and M06-2X) as well as the dispersion scheme vdW-TS produce energy barriers, $d_{O O}$ and $\delta$ parameters even smaller than optPBE-vdW, which lead us to conclude that NQC would result in a wider disagreement for the H-bond geometry compared to the experimental data.

On the other hand, the largest energy barriers, $d_{O O}$ and $\delta$ parameters are obtained with the non-local vdW functionals vdW-DF and vdW-DF2. The energy barrier in these cases is comparable in order of magnitude to the corresponding result for Möller-Plesset second order perturbation theory (MP2), which considers electron dynamical correlations, and hence van der Waals interactions, explicitly. A more accurate description of the effect of dynamical correlations on barriers and geometry would require either a coupled cluster calculation or the use of an RPA density functional, ${ }^{70}$ both of which are computationally too expensive for this size of simulation cell. The non-local vdW method vdW-DF2 gives too large $d_{O O}$ and $\delta$ compared to experiment when NQC are applied to the H-bond geometry in KDP. NQC applied to the vdW-DF geometry leads to a very good correspondence with the experiment for both the O-O distance and the parameter $\delta$ (see Table I). Moreover, the vdW-DF functional supplemented with NQC produces a remarkable agreement with the experiment also for the deuterated case as can be judged in Table III by comparing with the experimental results for DKDP. Therefore, unless we state the contrary, all the remaining calculations in this work are carried out using the ab initio method vdW-DF, which affords, after inclusion of NQC, the best performance for the geometric parameters of both KDP and DKDP.

In Table II we show the results of the structural parameters for the PE and FE phases of KDP obtained with the chosen AI method, vdW-DF. These results exhibit a good overall agreement with the experimental data. The shorter O-O distance in the $\mathrm{PE}$ phase can be attributed mainly to the static optimization for centered protons in 


\begin{tabular}{|c|c|ccc|c|}
\hline \hline DFT & Energy Barriers & & FE & & PE \\
Methods & $\Delta E n[m e V / f . u]$. & $d(O \cdots O)$ & $d(O-H)$ & $\delta$ & $d(O \cdots O)$ \\
\hline PBE (VASP) & $25[166]$ & $2.477(2.426)$ & $1.070(1.206)$ & $0.338(0.015)$ & 2.411 \\
PBE (QE) & 37 & 2.497 & 1.060 & 0.378 & 2.420 \\
PBE (CP2K) & 33 & 2.488 & 1.062 & 0.364 & 2.415 \\
DFT-D2 (VASP) & $25[167]$ & $2.478(2.426)$ & $1.068(1.204)$ & $0.341(0.017)$ & 2.410 \\
DFT-D2 (QE) & 38 & 2.493 & 1.061 & 0.372 & 2.416 \\
PBE0 (VASP) & 57 & 2.486 & 1.039 & 0.407 & 2.390 \\
PBE0-ADMM (CP2K) & 81 & 2.494 & 1.033 & 0.427 & 2.395 \\
VdW-TS (VASP) & 25 & 2.477 & 1.070 & 0.338 & 2.411 \\
vdW-DF (VASP) & $\mathbf{1 2 1}[\mathbf{5 2 8}]$ & $\mathbf{2 . 5 7 5}(\mathbf{2 . 5 0 6 )}$ & $\mathbf{1 . 0 2 8}(\mathbf{1 . 0 7 6})$ & $\mathbf{0 . 5 1 8}(\mathbf{0 . 3 5 5})$ & $\mathbf{2 . 4 2 4}$ \\
vdW-DF (QE) & 134 & 2.574 & 1.024 & 0.526 & 2.424 \\
vdW-DF2 (VASP) & $181[645]$ & $2.599(2.562)$ & $1.021(1.043)$ & $0.557(0.476)$ & 2.433 \\
vdW-DF2 (QE) & 188 & 2.599 & 1.018 & 0.563 & 2.432 \\
HSE06 (VASP) & 58 & 2.484 & 1.039 & 0.406 & 2.387 \\
HSE06 (QE) & 74 & 2.491 & 1.035 & 0.422 & 2.392 \\
optPBEvdW (VASP) & $77[365]$ & $2.539(2.446)$ & $1.043(1.186)$ & $0.454(0.074)$ & 2.422 \\
opt88vdW (VASP) & 63 & 2.509 & 1.058 & 0.393 & 2.421 \\
opt88vdW (QE) & 64 & 2.511 & 1.053 & 0.406 & 2.423 \\
M06-2X-ADMM (CP2K) & 80 & 2.492 & 1.039 & 0.414 & 2.397 \\
MP2 (CP2K) & 241 & 2.523 & 1.010 & 0.503 & 2.384 \\
\hline Expt. ${ }^{57}$ (KDP) & - & $\mathbf{2 . 4 9 7}$ & $\mathbf{1 . 0 5 6}$ & $\mathbf{0 . 3 8 5}$ & $\mathbf{2 . 4 8 3}$ \\
\hline \hline
\end{tabular}

Table I: Ab initio results for the global-displacement energy barriers and H-bond structural parameters in the PE and FE phases for several exchange-correlation functionals and DFT methods, calculated with VASP, QE and CP2K. Also shown are the experimental H-bond parameters of KDP for the FE and PE phases. Calculated H-bond parameters after NQC for KDP (see explanations in text) are shown in parenthesis. Notice that in the case of vdW-DF (VASP), the inclusion of quantum nuclear effects brings $d_{O O}, d_{O H}$ and $\delta$ much closer to experiment. We also show in brackets the energy barriers between the energy minimum and the transition state $(x=0)$ which were obtained in the FE phase by fixing the O-O distance. Distances are in $\AA$.

the H-bonds, ${ }^{3,38}$ while in the actual PE phase the protons are delocalized over two symmetric, off-center positions along the bond. In addition, we observe a slight overestimation in the calculated P-O distances when compared to the corresponding experimental values. This was also found in previous AI calculations in $\mathrm{H}$-bonded $\mathrm{FE}$ and AFE compounds. $3,38,71,72$

\section{B. Nuclear quantum effects in the H-bond geometry}

H-bonds suffer important structural changes upon deuteration. For instance, neutron diffraction experiments show an expansion of the $d_{O O}$ and $\delta$ parameters with deuteration, a phenomenon often referred to as geometrical or Ubbelohde effect. $6,57,73$ There is a strong correlation between $d_{O O}$ and $\delta$ which is regulated by nuclear quantum delocalization in a self-consistent way, a mechanism that was unveiled by ab initio calculations. ${ }^{3,15}$ In order to describe nuclear quantum and thermal effects in the $\mathrm{O}-\mathrm{H} \cdots \mathrm{O}$ bonds of KDP and DKDP, we develop, in this subsection, a simple, minimal, three-site linear model that captures the essential physics behind the selfconsistent phenomenon between geometry and tunneling. The model is adjusted to ab initio data and applied to the study of the geometrical effect at finite temperature. ${ }^{38}$ The NQC obtained for this model were used in Section III A to correct the first-principles calculations, which correspond to classical nuclei, i.e. having infinite mass, and zero temperature. This enables us to compare directly the corrected geometry with the experiment (see values in parenthesis in Tables I and II).

The $\mathrm{O}-\mathrm{H}$. . O bond is described by the following potential:

$$
V(x, R)=V_{O H}\left(x+\frac{R}{2}\right)+V_{O H}\left(\frac{R}{2}-x\right)+V_{O O}(R),
$$

where $x$ represents the position of the $\mathrm{H}$ atom measured from the center of the $\mathrm{O}-\mathrm{O}$ bond, and $R \equiv d_{O O}$ is the $\mathrm{O}-\mathrm{O}$ distance (see Fig. 1).

In Eq. (1), $V_{O H}$ is a Morse potential describing the $\mathrm{O}-\mathrm{H}$ interaction: 


\begin{tabular}{|c|ccc|ccc|}
\hline \hline $\begin{array}{c}\text { Structural } \\
\text { Parameters }\end{array}$ & Expt. & vdW-DF (VASP) & vdW-DF (QE) & Expt. & vdW-DF (VASP) & vdW-DF (QE) \\
\hline$d(O \cdots O)$ & 2.497 & $2.575(2.506)$ & 2.574 & 2.483 & 2.424 & 2.424 \\
$d(O 2-H)$ & 1.056 & $1.028(1.076)$ & 1.024 & 1.071 & 1.212 & 1.212 \\
$d(H \cdots O 1)$ & 1.441 & 1.546 & 1.550 & 1.412 & 1.212 & 1.212 \\
$\delta$ & 0.385 & $0.518(0.355)$ & 0.526 & & & 179.3 \\
$\angle(O 2-H \cdots O 1)$ & 179.4 & 178.5 & 178.7 & 178.2 & 179.4 & 3.466 \\
$d(P \cdots K)$ & - & 3.3 & 3.3 & - & 3.466 & 3.466 \\
$d(K \cdots P)$ & - & 3.596 & 3.583 & - & 3.466 & 1.561 \\
$d(P-O 1)$ & 1.516 & 1.522 & 1.520 & & & 2.811 \\
$d(P-O 2)$ & 1.572 & 1.608 & 1.610 & 1.543 & 1.561 & \\
$d(K \cdots O 1)(\mathrm{nn})$ & 2.785 & 2.734 & 2.741 & & & 2.810 \\
$d(K \cdots O 2)(\mathrm{nn})$ & 2.825 & 2.776 & 2.767 & 2.809 & & 2.886 \\
$d(K \cdots O 1)(\mathrm{nnn})$ & 2.847 & 2.851 & 2.866 & & & \\
$d(K \cdots O 2)(\mathrm{nnn})$ & 2.914 & 2.918 & 2.900 & 2.881 & 2.884 & \\
\hline \hline
\end{tabular}

Table II: Ab initio structural parameters for the PE and FE phases of KDP calculated with the XC-functional vdW-DF using VASP and QE. Also shown are the experimental data of Ref. ${ }^{57}$ for KDP. We show in parenthesis the values obtained after NQC. Distances are in $\AA$ and angles in degrees.

$$
V_{O H}(q)=D\left(1-e^{-a\left(q-r_{0}\right)}\right)^{2}-D
$$

while $V_{O O}$ is a Morse potential that represents the interaction between both oxygens and the lattice:

$$
V_{O O}(R)=D_{O O}\left(1-e^{-a_{O O}\left(R-R_{0}\right)}\right)^{2}-D_{O O}
$$

The sum of the first two terms in Eq. (1) corresponds to a back-to-back double-Morse potential acting on the hydrogen, which is commonly used for describing $\mathrm{H}-$ bonds. ${ }^{39,74-76}$ The anharmonic potential of Eq. (1) reflects the correlation between the $\mathrm{H}$ displacement $x$ and the O-O distance $R$ observed in experiments and ab initio calculations: when the $\mathrm{H}$ approaches one of the O's in the covalent bond $\mathrm{O}-\mathrm{H}$ (increasing $x$ ), the hydrogenbond with the other $\mathrm{O}$ weakens and the $\mathrm{O}-\mathrm{O}$ distance $(R)$ increases. The inverse situation also holds: $R$ diminishes with decreasing $x$. This correlation is precisely the important ingredient necessary for the existence of the Ubbelohde effect observed in compounds with strong H-bonds. Notice that the last term in Eq. (1), which represents the interaction of both oxygens with the lattice, is necessary in order to prevent the $\mathrm{H}$-bond to collapse into a single-minimum potential $(x=0)$ following structural optimization.

In order to describe nuclear quantum effects for the $\mathrm{H}-$ bond geometry, we perform Path Integral Monte Carlo (PIMC) simulations ${ }^{77}$ with the three-site model above defined. The model has six parameters to be adjusted, three from each one of the interaction potentials: $D, a$, and $r_{0}$ for $V_{O H}$, and $D_{O O}, a_{O O}$, and $R_{0}$ for $V_{O O}$. These parameters have been adjusted to reproduce six magnitudes obtained from the ab initio calculations. Four of these ab initio magnitudes to be reproduced are the global energy barrier between the PE and FE states, the $\mathrm{O}-\mathrm{O}$ and $\delta$ distances in the $\mathrm{FE}$ phase, and the $\mathrm{O}-\mathrm{O}$ distance in the PE phase (see Table I). The other two ab initio magnitudes are the energy barrier between the energy minimum and the transition state $(x=0)$ in the FE phase keeping the $\mathrm{O}-\mathrm{O}$ distance fixed (see values in brackets in Table I), and the ab initio vibrational frequency of the $\mathrm{PO}_{4}$ rotation mode $\left(\approx 543 \mathrm{~cm}^{-1}\right.$ in the vdW-DF case) (see Table $\mathrm{V}$ and Fig. 9). Ab initio results for this frequency are similar for the functionals indicated in Table III, e.g. the largest difference found between these values is $\approx 30 \mathrm{~cm}^{-1}$. The $\mathrm{PO}_{4}$-rotation mode in the KDP lattice is analogous to the symmetric stretching mode, which corresponds, in the three-site linear model, to the $\mathrm{O}-\mathrm{O}$ out-of-phase breathing mode around the $\mathrm{H}$ atom.

The mode frequencies are obtained by diagonalizing the $3 \times 3$ dynamical matrix constructed with the force constants of the three-site linear model. One of the three normal modes corresponds to the rigid translation, and has zero frequency. The other two modes correspond to symmetric and anti-symmetric stretching vibrations.

Model parameters were obtained by fitting to VASPcalculations with the following $\mathrm{XC}$ functionals: $\mathrm{PBE}$, DFT-D2, optPBEvdW, vdW-DF, and vdW-DF2 (see Table I). The best fits to ab initio results are reported in Table III.

For each ab initio method, PIMC calculations of the three-site model described by the bidimensional potential of Eq. (1) were performed using the corresponding parameter set obtained from the fit (see Table III). The effective masses used for $\mathrm{H}$ and $\mathrm{D}$ were $\mu_{H}=2.3 m_{p}$ and $\mu_{D}=3 m_{p}$, respectively, with $m_{p}$ the proton mass. The reason for not using the bare proton or deuteron masses 
$m_{p}$ and $2 m_{p}$ is that the motion of the proton/deuteron is strongly correlated with that of the heavy ions - $\mathrm{O}$ in this case -, and its mass is dressed accordingly as discussed in Ref. ${ }^{3}$.

In the PIMC simulations, the effective short-time propagator for two adjacent points in the discretized imaginary-time path describing each quantum particle is evaluated to fourth-order accuracy with the TakahashiImada approximation. ${ }^{77-79}$ The effective action in this case allows us to significantly reduce the Trotter number $M$ required for convergence. In all the simulations performed we have used $M=128$ beads for the quantum polymer associated to each atom in the $\mathrm{O}-\mathrm{H} \cdots \mathrm{O}$ bonds, which yielded well-converged results. ${ }^{38,77}$ Additionally, a normal-mode representation of the quantum polymers was used in order to ensure ergodicity in the MC sampling. ${ }^{77,79}$ The PIMC simulations were performed at $\mathrm{T}=102 \mathrm{~K}$ in order to compare the results to the experimental data measured at this temperature ${ }^{57}$, and consisted of $5 \times 10^{6} \mathrm{MC}$ steps preceeded by $5 \times 10^{5}$ steps of thermalization.

Results for the NQC applied to the H-bond geometry for KDP and DKDP are shown in Table III and compared to the corresponding experimental results in the FE phase. We conclude that, amongst the studied functionals, vdW-DF exhibits the best agreement with the experimental geometry for both KDP and DKDP. The isotope effect in the H-bond geometry calculated with this method follows the same trend as the experimental data in spite of the simplicity of the model. For instance, for the vdW-DF functional, the change in the O-O distance upon deuteration is $\approx+0.023 \AA$, which is found to be in rather good agreement with the corresponding change in the experimental data, $\approx+0.036 \AA$ (see Table III).

We would like to stress here that there is a subtle relation between quantum and thermal fluctuations which determines the H-bond geometry at low temperature. Without considering nuclear quantum effects, the $\mathrm{O}-\mathrm{O}$ distance and the volume of the system in the FE phase are found to increase modestly with temperature, as indicated by preliminary ab initio molecular dynamics simulations with the vdW-DF functional in KDP. When the quantum nature of the proton(deuteron) is taken into account, the proton(deuteron) delocalization acts as a glue between oxygens and produces a contraction of the OO distance, a mechanism which is deeply related to the huge isotope effect in KDP. ${ }^{3,15}$ This mechanism is enhanced when the temperature increases as is observed in our model simulations at low temperature. For instance, we find a contraction of the $\mathrm{O}-\mathrm{O}$ bond mediated by the proton delocalization with increasing temperature: the $\mathrm{O}-\mathrm{O}$ distance is reduced from $\approx 2.530 \AA$ at $50 \mathrm{~K}$ to $\approx 2.506 \AA$ at $102 \mathrm{~K}$ (the $\mathrm{O}-\mathrm{O}$ contraction is $\approx 0.024$ $\AA$ ). However the contraction with temperature, due to its quantum nature, is less pronounced in the deuterated case: e.g. the $\mathrm{O}-\mathrm{O}$ distance shortens from $\approx 2.549 \AA$ at $50 \mathrm{~K}$ to $\approx 2.529 \AA$ at $102 \mathrm{~K}$ (here the contraction am- mounts to $\approx 0.020 \AA$ ). As a consequence, the increase in the $\mathrm{O}-\mathrm{O}$ distance with deuteration (Ubbelohde effect) is slightly smaller at $50 \mathrm{~K}(\approx+0.019 \AA)$ than at $102 \mathrm{~K}$ $(\approx+0.023 \AA)$. Interestingly, a volume contraction with temperature is observed in normal and deuterated ice at low temperature, as well as an anomalous lattice expansion with deuteration. ${ }^{80,81}$ These features for the H-bond geometry of ice are similar to what we found here for KDP with the PIMC simulations of a simple three-site model adjusted to ab initio results, suggesting that this behavior might be related to local and general properties of the H-bonds which are independent of the bridges environment.

The diagonalization of the dynamical matrix for the three-site linear model also gives the frequency of the anti-symmetric stretching mode. This mode, which has the largest frequency in the model, corresponds to the stretching of the $\mathrm{O}-\mathrm{H}$ bond. The frequency of this mode in the model fitted to the vdW-DF ab initio data is 3186 $\mathrm{cm}^{-1}$, which is significantly larger than typical frequencies of O-H stretching modes in the actual KDP system, $\approx 2600 \mathrm{~cm}^{-1}$ (see Tables $\mathrm{V}$ and VI). This is probably due to an overestimation of the repulsive forces for the simulated covalent bonds as well as to the simplifications in the linear-molecule model. The quantum (PIMC) calculation leads to a variation of the molecular geometry, and thus to a renormalization of the mode frequencies. Hence, we rebuild the dynamical matrix of the model after NQC with the quantum-corrected $\mathrm{O}-\mathrm{O}$ and $\mathrm{O}-\mathrm{H}$ distances (see Table III) and, after a new diagonalization with the corrected geometry, the anti-symmetric stretching frequency changes to $2630 \mathrm{~cm}^{-1}$ (KDP) and 2557 $\mathrm{cm}^{-1}$ (DKDP). This may appear counterintuitive at first glance, since purely on the basis of isotopic substitution in a fixed potential energy surface (PES), we would expect a larger renormalization for the protonated system. Here, however, frequencies are calculated assuming a harmonic PES via frozen-phonon calculations. Therefore, in the present calculations, the isotope effect in the frequencies arises from geometric variations (Ubbelohde effect), and different effective masses due to coupling with the heavy atoms. Deuteration shortens the O-D distance as shown in Table III and repulsive forces in the O-D bond are stronger, thus leading to a larger force constant in the deuterated system. This, however, is counteracted by the larger effective mass in the deuterated system. Since frequencies are given by the ratio between force constant and effective mass, the combination of geometric and mass effects is quite similar for the protonated and deuterated systems, thus leading to only minor differences in frequency. Infrared measurements show a very small strongly-damped proton-stretching band that extends up to a maximum frequency of $2500 \mathrm{~cm}^{-1} .{ }^{18}$ This frequency limit is in good agreement with our predicted anti-symmetric stretching frequency obtained when NQC are applied to the KDP case. 


\begin{tabular}{|c|ccccc|ccccccc|}
\hline \hline DFT & \multicolumn{4}{|c|}{ Parameters } & \multicolumn{3}{c|}{ KDP $\left(\mu_{H}=2.3 m_{p}\right)$} & \multicolumn{3}{c|}{ DKDP $\left(\mu_{D}=3.0 m_{p}\right)$} \\
Methods & $D[\mathrm{eV}]$ & $a\left[\AA^{-1}\right]$ & $r_{0}[\AA]$ & $D_{O O}[\mathrm{eV}]$ & $a_{O O}\left[\AA^{-1}\right]$ & $R_{0}[\AA]$ & $d(O \cdots O)$ & $d(O-H)$ & $\delta$ & $d(O \cdots O)$ & $d(O-D)$ & $\delta$ \\
\hline PBE & 16.621 & 2.761 & 0.949 & 45.103 & 0.594 & 2.883 & 2.426 & 1.206 & 0.015 & 2.425 & 1.204 & 0.018 \\
DFT-D2 & 15.709 & 2.786 & 0.951 & 79.522 & 0.478 & 2.849 & 2.426 & 1.204 & 0.017 & 2.424 & 1.204 & 0.017 \\
vdW-DF & 8.838 & 3.027 & 0.966 & 10.542 & 0.831 & 2.917 & $\mathbf{2 . 5 0 6}$ & $\mathbf{1 . 0 7 6}$ & $\mathbf{0 . 3 5 5}$ & $\mathbf{2 . 5 2 9}$ & $\mathbf{1 . 0 5 9}$ & $\mathbf{0 . 4 1 1}$ \\
vdW-DF2 & 7.819 & 3.125 & 0.969 & 3.894 & 1.116 & 2.951 & 2.562 & 1.043 & 0.476 & 2.570 & 1.038 & 0.492 \\
optPBEvdW & 10.080 & 2.963 & 0.964 & 9.034 & 0.897 & 2.934 & 2.446 & 1.186 & 0.074 & 2.465 & 1.114 & 0.237 \\
\hline Expt. $^{57}$ & & & & & & & $\mathbf{2 . 4 9 7}$ & $\mathbf{1 . 0 5 6}$ & $\mathbf{0 . 3 8 5}$ & $\mathbf{2 . 5 3 3}$ & $\mathbf{1 . 0 3 1}$ & $\mathbf{0 . 4 7 2}$ \\
\hline \hline
\end{tabular}

Table III: Nuclei quantum calculations of the H-bond geometries for KDP and DKDP using the three-site linear model adjusted to the ab-initio VASP calculations for different XC functionals (see also Table I). The results are contrasted with the experimental data of Ref. ${ }^{57}$. Also shown are the corresponding parameters set of the model which were fit for each calculation (see explanations in text). Distances are in $\AA$.

\section{PHONONS AND RELATED PROPERTIES}

\section{A. Symmetry of the normal modes at the $\Gamma$-point}

The complete symmetry analysis of the phonons in KDP has been done long ago by Shur for the PE and FE phases, and for all the high-symmetry points in the Brillouin zone. ${ }^{82}$ The symmetries of the $\Gamma$-point modes are shown in Table IV, where the infrared (IR) and Raman (R) activity is also indicated. Only the IR active phonons can induce polarization. For instance, in the $\mathrm{PE}$ phase the non-degenerate $\mathrm{B}_{2}$ phonons induce polarization along the $c$-axis, while the two-fold degenerate $\mathrm{E}$ phonons are polarized perpendicular to that axis.

\begin{tabular}{cccc}
\multicolumn{4}{c}{ Paraelectric } \\
\hline \hline Symmetry & Activity & $\mathrm{m}_{j}$ & $\mathrm{~d}_{j}$ \\
\hline $\mathrm{A}_{1}$ & $\mathrm{R}$ & 4 & 1 \\
$\mathrm{~A}_{2}$ & - & 5 & 1 \\
$\mathrm{~B}_{1}$ & $\mathrm{R}$ & 6 & 1 \\
$\mathrm{~B}_{2}$ & $\mathrm{IR}, \mathrm{R}$ & 7 & 1 \\
$\mathrm{E}$ & $\mathrm{IR}, \mathrm{R}$ & 13 & 2 \\
\hline \hline
\end{tabular}

\begin{tabular}{cccc}
\multicolumn{4}{c}{ Ferroelectric } \\
\hline \hline Symmetry & Activity & $\mathrm{m}_{j}$ & $\mathrm{~d}_{j}$ \\
\hline $\mathrm{A}_{1}$ & $\mathrm{IR}, \mathrm{R}$ & 11 & 1 \\
$\mathrm{~A}_{2}$ & $\mathrm{R}$ & 11 & 1 \\
$\mathrm{~B}_{1}$ & $\mathrm{IR}, \mathrm{R}$ & 13 & 1 \\
$\mathrm{~B}_{2}$ & $\mathrm{IR}, \mathrm{R}$ & 13 & 1 \\
\hline \hline
\end{tabular}

Table IV: Summary of the mode symmetries at the $\Gamma$-point for the paraelectric and ferroelectric phases. In the first column the irreducible representations are listed. $\mathrm{R}$ and IR indicate respectively Raman and Infrared activity. $m_{j}$ indicates the number of modes in each representation and $d_{j}$ their degeneracy.

A group theoretical analysis provides the correspondence between irreducible representations in the $\mathrm{PE}$ phase (space group $\mathrm{I} \overline{4} 2 \mathrm{~d}$ or $\mathrm{D}_{2 d}^{12}$ ) and the FE phase (space group Fdd2 or $\left.\mathrm{C}_{2 v}^{19}\right) \cdot{ }^{22}$ Due to the change from tetrago- nal to orthorhombic symmetry, the doubly-degenerate $\mathrm{E}$ modes split into modes of symmetries $\mathrm{B}_{1}$ and $\mathrm{B}_{2}$ (see table IV). The reduction in symmetry originated in the off-centering of the protons leads to the collapse of representations $\mathrm{A}_{1}$ and $\mathrm{B}_{2}$ onto $\mathrm{A}_{1}$, and of $\mathrm{A}_{2}$ and $\mathrm{B}_{1}$ onto $\mathrm{A}_{2}$.

B. Ab initio results for the $\Gamma$-point phonons, phonon dispersion curves and phonon density of states

The ab initio vibrational frequencies of KDP at the $\Gamma$-point of the Brillouin zone obtained with the vdW-DF (VASP) functional are reported in Tables V and VI, and compared to the available experimental data. The tables are organized according to the correspondence between representations in the $\mathrm{PE}$ and $\mathrm{FE}$ phases explained in the previous subsection.

In Table $\mathrm{V}$, the second column in the left panel contains the ab initio frequencies of the $\mathrm{A}_{1}, \mathrm{~A}_{2}, \mathrm{~B}_{1}$ and $\mathrm{B}_{2}$ modes in the $\mathrm{PE}$ phase, divided into the two subspaces, $\left(\mathrm{A}_{1}+\right.$ $\left.\mathrm{B}_{2}\right)$ and $\left(\mathrm{A}_{2}+\mathrm{B}_{1}\right)$. In the second column of the right panel we report the corresponding ab initio frequencies of the $A_{1}$ and $A_{2}$ modes in the FE phase. Frequencies in the same row correspond in general to qualitatively similar patterns of atomic motion in the normal modes in both phases, although in some cases the comparison between patterns was not straightforward. Therefore, the correspondence between modes in the two phases has to be taken with caution. Similarly, in Table VI we show, in the second column of the left panel, the ab initio frequencies of the phonons with E symmetry in the PE phase. In the second column in the right panel we report the $\mathrm{B}_{1}$ and $\mathrm{B}_{2}$ ab initio frequencies in the $\mathrm{FE}$ phase split from every doubly-degenerate $\mathrm{E}$ mode in the PE phase.

We have also calculated the phonon dispersion curves for the FE phase along the high-symmetry directions of the Brillouin zone, using the vdW-DF (VASP) functional, and the FD approach as implemented in the PHONOPY package (see explanation in Section II). The total and partial (where the contributions of the different atoms, $\mathrm{K}, \mathrm{P}, \mathrm{O}, \mathrm{H}$, is discriminated) phonon density of states 
(DOS) were also derived from the ab initio phonon calculations with the help of the PHONOPY code. The phonon dispersion curves, as well as, the total and partial DOS in the FE phase are shown in Fig. 2.

\section{Calculated phonons at the $\Gamma$-point and phonon bands}

We observe in both, Table V and Table VI, a good agreement between our ab initio frequencies for the $\Gamma$ point phonons and the available experimental data.

Three unstable modes, identified by their imaginary frequencies, arise in the PE phase. These are stabilized in the FE phase, becoming high-frequency $\mathrm{OH}-$ stretching modes (see Tables V and VI. See also the highest frequency band at $\approx 2600 \mathrm{~cm}^{-1}$ corresponding to the stretching H-modes in Fig. 2). One of these three is precisely the $\mathrm{B}_{2} \mathrm{FE}$ soft-mode which leads to the spontaneous polarization of the crystal along the $c$-axis below the FE transition temperature. ${ }^{3,32}$ The largest displacements in this mode are those of the hydrogen atoms along the O-H-O bonds, i.e. in a direction almost perpendicular to the $c$-axis. As depicted in Fig. 3, the pattern of these displacements consists of two $\mathrm{H}$ atoms approaching the bottom oxygens $\mathrm{O}_{2}$ of the $\mathrm{PO}_{4}$ tetrahedron, while the other two $\mathrm{H}$ atoms move away from the top oxygens $\mathrm{O}_{1}$. In this way, the ice rules are fulfilled. Concomitantly, the $\mathrm{P}$ atoms move, in a smaller amount, along the $z$-axis towards the top oxygens $\mathrm{O}_{1}$, resulting in a distortion and polarization of the phosphates in the FE phase (see Table II). Finally, the $\mathrm{K}^{+}$ions displace along $z$ towards the negatively charged side of the $\mathrm{PO}_{4}$ molecule (i.e. that of the $\mathrm{O}_{1}$ atoms), but in a much smaller proportion. The whole eigenvector pattern coincides quite well with the one reported in a previous theoretical (non ab initio) calculation, ${ }^{30}$ and also in experimental works. ${ }^{18,57}$

The other two unstable modes observed in the PE phase correspond to a two-fold degenerate E-mode polarized perpendicularly to the $c$-axis (see Table VI) ${ }^{32}$ Its pattern is shown in Fig. 4. Also in this case the largest displacements are those of the hydrogen atoms and, as for the FE-mode, only two hydrogens move simultaneously towards each $\mathrm{PO}_{4}$ tetrahedron, thus fulfilling the ice rules. In this case, one $\mathrm{H}$-atom approaches the oxygen at the top of the tetrahedron $\left(\mathrm{O}_{1}\right)$, while the other $\mathrm{H}$-atom approaches the oxygen at the bottom $\left(\mathrm{O}_{2}\right)$. In these modes the $\mathrm{P}$ atoms displace along the basal plane towards the negatively-charged lateral oxygens polarizing the $\mathrm{PO}_{4}$ tetrahedron in the $x y$-plane. Four equivalent patterns can be formed in this way, which are identified as the four lateral Slater (LS) configurations for the $\mathrm{H}_{2} \mathrm{PO}_{4}$ group. ${ }^{85}$ According to first-principles calculations, ${ }^{86}$ strong correlations among LS defects are present in the system leading to the stabilization of long chains of defects. The energy per phosphate of these correlated defects is only $\approx 5 \mathrm{meV} /$ formula-unit higher in energy than the corresponding value for the FE polar mode. This value is in good agreement with that deduced from model calculations. ${ }^{87}$

A strongly damped asymmetric peak in the lowfrequency range was observed in the infrared spectrum, polarized perpendicularly to the $c$-axis for both KDP and DKDP by Bréhat and Wyncke ${ }^{29,88}$. This signature, produced by the lowest-frequency transverse optical (TO) mode, is associated to a proton collective motion of $\mathrm{E}$ symmetry which causes the fluctuation of polarization in the $x y$-plane. The observed peak disappears in the lowfrequency region of the ferroelectric phase. These experimental findings enable us to link the infrared feature to the LS instabilities associated to the $\mathrm{E}$ phonons found in our ab initio calculations (see Table VI). This mode was also predicted within the modified strong dipole-proton coupling model of Merunka and Rakvin. ${ }^{89}$

Interestingly, the three unstable phonons in the $\mathrm{PE}$ phase correspond to the energetically most favorable configurations in the Slater-Takagi picture. ${ }^{85,90}$ The most energetic Slater-Takagi configuration in this picture has the four $\mathrm{H}$-atoms approaching the $\mathrm{PO}_{4}$ tetrahedron simultaneously. This configuration violates the ice rules, but it is certainly possible. In fact, we find this hydrogen breathing pattern in the highest frequency $\mathrm{A}_{2}$ mode at $2719 \mathrm{~cm}^{-1}$ in the FE phase (see Table V). A similar $\mathrm{A}_{2}$ mode is observed at $1104 \mathrm{~cm}^{-1}$ in the PE phase. Other Takagi defects characterized by one or three $\mathrm{H}$ atoms approaching the phosphate ions, are not present as $\Gamma$-point phonons as the motion pattern extends beyond the unit cell. Consequently, they appear at other points in the Brillouin zone.

The modes corresponding to the Slater-Takagi configurations mentioned above are precisely the $\mathrm{H}$-stretching (HS) vibrations which belong to the highest frequency band in the region between $\approx 2450$ and $\approx 2750 \mathrm{~cm}^{-1}$ in the FE phonon spectrum (see Fig. 2). These frequencies are the highest ones for two reasons: i) the stiffness of the covalent $\mathrm{O}-\mathrm{H}$ bond, and ii) the fact that the modes involve primarily the motion of the lightest atom $(\mathrm{H})$. However, if the hydrogen atoms oscillate perpendicular to the H-bonds, the resulting force constants are comparatively smaller. Phonons with these patterns, i.e. Hbending (HB) modes, have lower frequencies. Indeed, pure HB modes can be identified with a dispersion-less branch at $\approx 1300 \mathrm{~cm}^{-1}$ in the FE phase, as shown in Fig. 2. A large gap of $\approx 1050 \mathrm{~cm}^{-1}$ separate this band from the HS one. A HB band centered at $\approx 1000 \mathrm{~cm}^{-1}$ is also observed in the FE phase, although in this case it is considerably mixed with the internal-molecular (IM) phosphate modes with $\mathrm{O}$ and $\mathrm{P}$ displacements (see the partial DOS in Fig. 2). This indicates that, contrary to the HS modes, HB phonons soften in going from the PE to the FE phase (see Tables V and VI). This can be understood by noticing that, in the PE phase, the $\mathrm{H}$ located in the center of the O-H-O bridge is strongly bound to both oxygens, thus increasing the stiffness of the bending potential. This stiffness is partially lost in the FE phase with the weakening of one of the covalent bonds.

Internal molecular (IM) phonons involving primarily 


\begin{tabular}{|c|c|c|c|c|c|c|c|c|c|c|c|}
\hline \multicolumn{6}{|c|}{ PE structure } & \multicolumn{6}{|c|}{ FE structure } \\
\hline Sym. & vdW-DF & Expt. $^{83}$ & Expt. ${ }^{24}$ & Expt. $^{26}$ & Class. & Sym. & vdW-DF & Expt. $^{83}$ & Expt. ${ }^{25}$ & Expt. ${ }^{18}$ & Class. \\
\hline B2 & $819 \mathrm{i}$ & & & & $\mathrm{FE}, \mathrm{HS}+\mathrm{IMB}$ & A1 & 2600 & & & & HS \\
\hline $\mathrm{B} 2$ & 189 & 180 & 180 & 179.5 & $\mathrm{ET}$ & A1 & 185 & 185 & 142 & 209 & ET \\
\hline A1 & 543 & & & 520.3 & IMB & A1 & 257 & & 283 & & IMB \\
\hline A1 & 295 & 360 & 363 & 363.9 & IMB & A1 & 345 & 346 & 369 & & IMB \\
\hline B2 & 391 & 395 & 393 & 394.0 & IMB & A1 & 383 & 394 & 393 & & IMB \\
\hline B2 & 533 & & & & IMB & A1 & 488 & 515 & & 440 & IMB \\
\hline A1 & 917 & 915 & & 916.9 & $\mathrm{IMS}+\mathrm{HS}$ & A1 & 833 & & & 859 & $\mathrm{IMS}+\mathrm{HB}$ \\
\hline $\mathrm{B} 2$ & 1145 & & & & $\mathrm{IMS}+\mathrm{HB}$ & A1 & 941 & 910 & & & $\mathrm{IMS}+\mathrm{HB}$ \\
\hline B2 & 1273 & & & & HB & A1 & 1002 & 1035 & & 1048 & $\mathrm{HB}+\mathrm{IMS}$ \\
\hline A1 & 1340 & & & & $\mathrm{HB}$ & A1 & 1282 & & & & HB \\
\hline B1 & 117 & & & & ET & $\mathrm{A} 2$ & 120 & & & & ET \\
\hline B1 & 174 & 152 & 151 & 155.8 & ET & $\mathrm{A} 2$ & 179 & 154 & 159 & & $\mathrm{ET}$ \\
\hline $\mathrm{A} 2$ & 224 & & & & ER & $\mathrm{A} 2$ & 221 & 206 & 211 & & ER \\
\hline $\mathrm{A} 2$ & 347 & & & & IMB & $\mathrm{A} 2$ & 354 & & & & IMB \\
\hline B1 & 475 & 475 & 470 & 474.5 & IMB & A2 & 467 & 485 & 483 & & IMB \\
\hline B1 & 680 & & & 564.1 & $\mathrm{IMB}+\mathrm{HS}$ & $\mathrm{A} 2$ & 529 & & & & $\mathrm{IMB}+\mathrm{HS}$ \\
\hline $\mathrm{A} 2$ & 826 & & & & $\mathrm{IMS}+\mathrm{HS}$ & A2 & 804 & & & & $\mathrm{IMS}+\mathrm{HB}$ \\
\hline B1 & 1021 & & & & $\mathrm{IMS}+\mathrm{HS}$ & $\mathrm{A} 2$ & 1027 & 1008 & & & $\mathrm{IMS}+\mathrm{HB}$ \\
\hline A2 & 1104 & & & & $\mathrm{HS}+\mathrm{HB}$ & $\mathrm{A} 2$ & 2719 & & & & HS \\
\hline B1 & 1349 & & & & HB & $\mathrm{A} 2$ & 1282 & & & & HB \\
\hline $\mathrm{A} 2$ & 1277 & & & & HB & $\mathrm{A} 2$ & 957 & & & & HB \\
\hline
\end{tabular}

Table V: Ab initio results of the Gamma-point phonons of symmetries A1,B2,A2,B1 (PE phase) and A1,A2 (FE phase) of KDP using VASP with the chosen XC-functional vdW-DF. Also shown are the experimental results of Refs. ${ }^{24,26,83}$ for the PE phase and Refs. ${ }^{18,25,83}$ for the FE phase. The mode-frequencies are shown in $\mathrm{cm}^{-1}$. According to the calculated eigenvectors, the following classification is shown in the table: ferroelectric unstable mode with imaginary frequency (FE), external traslational (ET), external rotational (ER), internal molecular P-O bending (IMB), internal molecular P-O stretching (IMS), $O-H \cdots O$ bending (HB), and $O-H \cdots O$ stretching (HS).

the $\mathrm{PO}_{4}$ tetrahedron are grouped in two bands in the $\mathrm{FE}$ phase, as shown in Fig. 2. The highest-energy one is located between 750 and $1200 \mathrm{~cm}^{-1}$ and consists of modes involving mainly the stretching of the P-O bonds (see also the corresponding zone-center phonons labeled as IMS: internal molecular stretching, in Tables V and VI). The other IM band spreads out in the region 300-600 $\mathrm{cm}^{-1}$, approximately, and comprises the O-P-O bending modes (see Fig. 2, and also the corresponding zone-center phonons labeled as IMB: internal molecular bending, in Tables $\mathrm{V}$ and VI). The O-P stretching (IMS) phonons can be of the breathing or quadrupolar type. In the latter, the $\mathrm{P}$ atoms oscillate out-of-phase with the oxygens. As mentioned before, the O-P stretching (IMS) band exhibits a considerable admixture with the $\mathrm{H}$-bending band around $1000 \mathrm{~cm}^{-1}$ in the FE phase. The IMS modes in the $\mathrm{PE}$ phase also have $\mathrm{H}$ stretching and bending displacements to some extent. The loss of rigidity in the H-bending displacements in going from the PE to the FE phase may explain the slight softening of the IMS phonons in this situation, as observed in Tables V and VI. On the other hand, the behavior of the O-P-O bending (IMB) modes across the phase transition is more subtle and difficult to rationalize, as some modes soften while others stiffen.

Vibrations below $300 \mathrm{~cm}^{-1}$ constitute a band that involves the motion of the $\mathrm{K}$ atoms (see Fig. 2). These phonons are lattice external translational (ET) and rotational (ER) modes which are generally fairly insensitive to the phase change as observed in Tables V and VI. Raman measurements in the $\mathrm{PE}$ phase of $\mathrm{KDP}^{24}$ report a missing optical lattice $\mathrm{B}_{1}$ mode. It has been speculated in Ref. ${ }^{24}$ that this missing phonon could have a very weak intensity or a very small frequency (less than $4 \mathrm{~cm}^{-1}$ ). Our calculations support the former case, since we have obtained a $\mathrm{B}_{1}$ ET mode of $117 \mathrm{~cm}^{-1}$ which has not been observed yet ${ }^{24,26,83}$ (see Table V) and falls in the frequency domain studied in Ref. ${ }^{24}\left(20-700 \mathrm{~cm}^{-1}\right)$.

\section{Comparison with experimental data}

Infrared reflectivity measurements have shown the softening of a $\mathrm{B}_{2}$ mode in the region around $440 \mathrm{~cm}^{-1}$ by about $60 \mathrm{~cm}^{-1}$ across the phase transition, and assigned it to the $\nu_{4}$ internal mode of the $\mathrm{PO}_{4}$ tetrahedron with 


\begin{tabular}{|c|c|c|c|c|c|c|c|c|c|c|}
\hline \multicolumn{6}{|c|}{ PE structure } & \multicolumn{5}{|c|}{ FE structure } \\
\hline Sym. & vdW-DF & Expt. $^{83}$ & Expt. $^{24}$ & Expt. $^{26}$ & Class. & Sym. & vdW-DF & Expt. $^{83}$ & Expt. ${ }^{18}$ & Class. \\
\hline $\mathrm{E}$ & $809 \mathrm{i}$ & & & & LS, HS + IMB & B1 & $2601(140)$ & & & HS \\
\hline $\mathrm{E}$ & $809 \mathrm{i}$ & & & & LS, HS + IMB & $\mathrm{B} 2$ & $2594(153)$ & & & HS \\
\hline $\mathrm{E}$ & 71 & 96 & 96 & 98.4 & ER & B1 & $106(2)$ & 95 & 110 & ER \\
\hline $\mathrm{E}$ & 72 & & & & ER & $\mathrm{B} 2$ & 124 & 100 & & ER \\
\hline $\mathrm{E}$ & 131 & 114 & 114 & 114.0 & ET & B1 & 138 & 140 & & ET \\
\hline $\mathrm{E}$ & 133 & & & & $\mathrm{ET}$ & $\mathrm{B} 2$ & $161(12)$ & & & $\mathrm{ET}$ \\
\hline $\mathrm{E}$ & 140 & & & & ET & B1 & $157(12)$ & & & $\mathrm{ET}$ \\
\hline $\mathrm{E}$ & 140 & & & & $\mathrm{ET}$ & B2 & 130 & & & $\mathrm{ET}$ \\
\hline $\mathrm{E}$ & 205 & 185 & 190 & 190.0 & ER & B1 & $213(24)$ & 220 & 213 & ER \\
\hline $\mathrm{E}$ & 205 & & & & $\mathrm{ER}$ & B2 & $194(4)$ & 180 & 192 & $\mathrm{ER}$ \\
\hline $\mathrm{E}$ & 383 & & & & IMB & B1 & $232(18)$ & & & IMB \\
\hline $\mathrm{E}$ & 383 & & & & IMB & B2 & $270(27)$ & 240 & & IMB \\
\hline $\mathrm{E}$ & 529 & 530 & 530 & 533.0 & $\mathrm{IMB}+\mathrm{HB}$ & B1 & $549(2)$ & 565 & & $\mathrm{IMB}+\mathrm{HB}$ \\
\hline $\mathrm{E}$ & 529 & 565 & & 564.1 & $\mathrm{IMB}+\mathrm{HB}$ & B2 & $472(12)$ & 543 & 480 & $\mathrm{IMB}+\mathrm{HB}$ \\
\hline $\mathrm{E}$ & 667 & & & & $\mathrm{IMB}+\mathrm{HS}$ & B1 & $502(4)$ & 517 & & $\mathrm{IMB}+\mathrm{HB}$ \\
\hline $\mathrm{E}$ & 667 & & & & $\mathrm{IMB}+\mathrm{HS}$ & B2 & $504(48)$ & 588 & 557 & $\mathrm{IMB}+\mathrm{HB}$ \\
\hline $\mathrm{E}$ & 1031 & & & & $\mathrm{IMS}+\mathrm{HS}$ & B1 & 879 & & 906 & $\mathrm{IMS}+\mathrm{HB}$ \\
\hline $\mathrm{E}$ & 1031 & & & & $\mathrm{IMS}+\mathrm{HS}$ & B2 & $856(36)$ & & 962 & $\mathrm{IMS}+\mathrm{HB}$ \\
\hline $\mathrm{E}$ & 1128 & 1120 & & & IMS + HS & B1 & $1081(5)$ & 1075 & 1154 & $\mathrm{IMS}+\mathrm{HB}$ \\
\hline $\mathrm{E}$ & 1128 & & & & $\mathrm{IMS}+\mathrm{HS}$ & B2 & $1088(17)$ & 1140 & & $\mathrm{IMS}+\mathrm{HB}$ \\
\hline $\mathrm{E}$ & 1291 & & & & HB & B1 & $1003(65)$ & & 1034 & HB \\
\hline $\mathrm{E}$ & 1291 & & & & HB & B2 & $1007(3)$ & & & $\mathrm{HB}$ \\
\hline $\mathrm{E}$ & 1363 & & & & HB & B1 & $1303(28)$ & & 1325 & $\mathrm{HB}$ \\
\hline $\mathrm{E}$ & 1363 & & & & $\mathrm{HB}$ & B2 & $1318(5)$ & & & HB \\
\hline
\end{tabular}

Table VI: Ab initio results of the Gamma-point phonons of symmetries E (PE phase) and B1,B2 (FE phase) of KDP using VASP with the chosen XC-functional vdW-DF. Also shown are the experimental results of Refs. ${ }^{24,26,83}$ for the PE phase and Refs. ${ }^{18,83}$ for the FE phase. The mode-frequencies are shown in $\mathrm{cm}^{-1}$. We also show in parenthesis the corresponding LO-TO splittings. According to the calculated eigenvectors, the following classification is shown in the table: lateral Slater unstable mode with imaginary frequency (LS), external traslational (ET), external rotational (ER), internal molecular P-O bending (IMB), internal molecular P-O stretching (IMS), $O-H \cdots O$ bending (HB), and $O-H \cdots O$ stretching (HS)

quadrupole phosphate distortion. ${ }^{18}$ In our calculations this is an IM O-P bending (IMB) mode that appears at the somewhat higher frequency of $533 \mathrm{~cm}^{-1}$ and softens to a frequency of $488 \mathrm{~cm}^{-1}$ in the FE phase (see bottom of Figure 8). This is one of the modes that couples strongly to the FE soft-mode, as will be discussed later.

The most significant frequency change between both phases is observed for the $\mathrm{A}_{1}$ mode at $543 \mathrm{~cm}^{-1}(\mathrm{PE}$ phase) which softens to $257 \mathrm{~cm}^{-1}$ in the FE phase (see Table V). This is precisely the mode that couples very strongly to the FE-mode as will be shown below. This mode can be described as rotations (librations) of adjacent $\mathrm{PO}_{4}$ tetrahedra around the $c$-axis in opposite directions, accompanied by bendings of the O-H-O bond (see top panel in Fig. 9). The reason for such a dramatic softening is quite clear. By off-centering the H-atoms in the $\mathrm{FE}$ phase, the $\mathrm{H}_{2} \mathrm{PO}_{4}$ groups are more weakly bound than those in the $\mathrm{PE}$ phase where the $\mathrm{H}$ atoms are centered. Hence, the $\mathrm{PO}_{4}$ tetrahedra can librate more freely and independently from the neighboring ones with the consequence of a reduction in the frequency of this motion.

To summarize the results of the AI DOS calculations in the FE phase, as a general behavior we observe four vibrational bands organized as follows: (i) a HS band between $\approx 2450$ and $2750 \mathrm{~cm}^{-1}$, (ii) a HB band in the region $\approx 1200-1400 \mathrm{~cm}^{-1}$, (iii) a "IMS + HB" band which extends from $\approx 750$ to $\approx 1170 \mathrm{~cm}^{-1}$, and (iv) a "E + IMB" band in the low-frequency region up to $\approx 620 \mathrm{~cm}^{-1}$ (see Fig. 2). This classification is in accordance with the obtained atomic partial DOS, which is shown in Fig. 2, and agrees with the assignment resulting from the eigenvector analysis of zone-center phonons (see the modes classification in Tables V and VI). The highest frequency bands, $\mathrm{HS}$ and $\mathrm{HB}$, are predominantly of $\mathrm{H}$ character, with a very small admixture of oxygen in the former, and oxygen and phosphorus in the latter. On the other hand, the "IMS + HB" and "E + IMB" bands have a considerable 

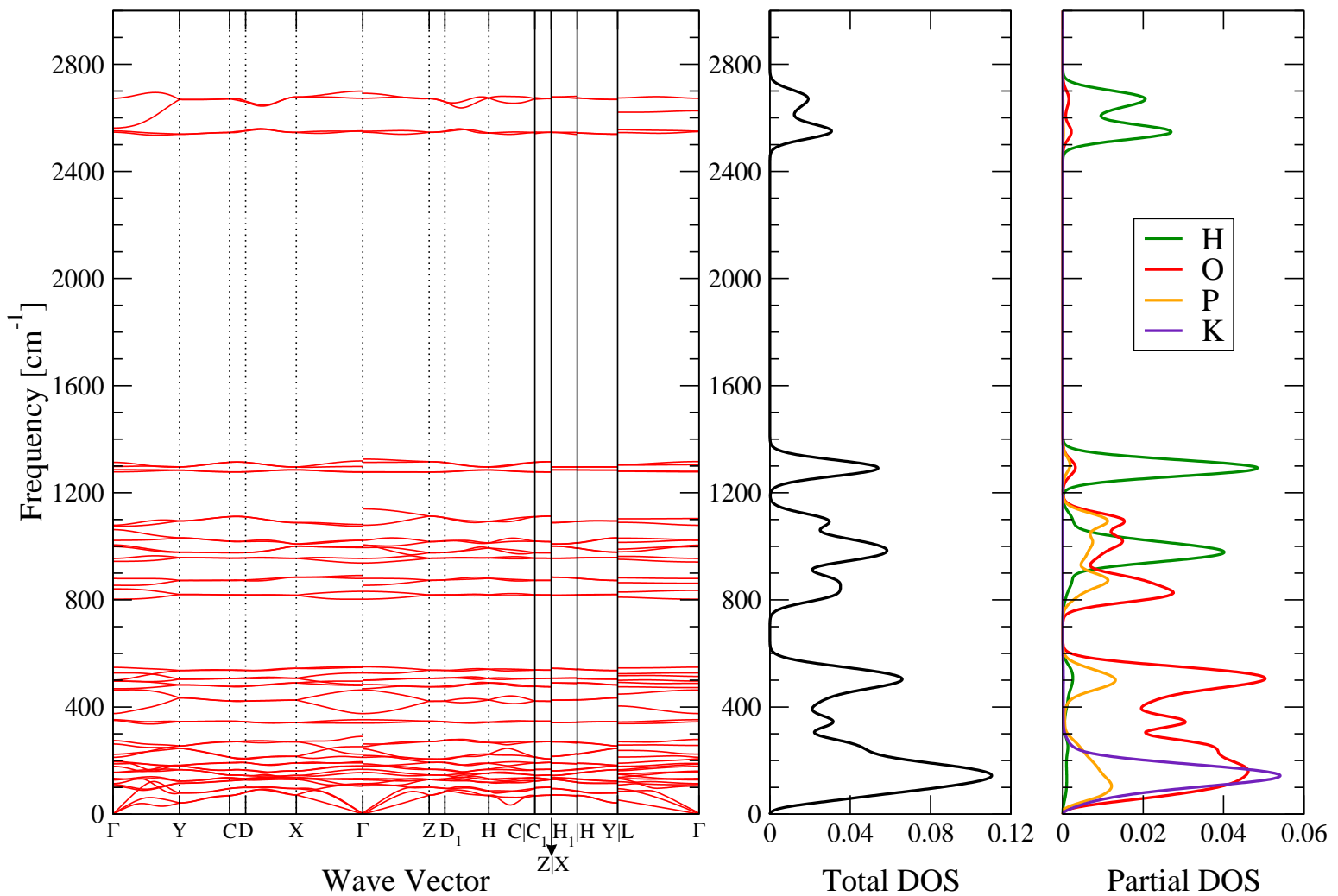

Figure 2: Ab initio results of the phonon dispersion curves in the FE phase of KDP. Also shown are the total and partial phonon density of states (DOS). The path in the Brillouin zone used for the phonon dispersion calculations corresponds to that reported in Fig. 10 of Ref. ${ }^{84}$ for the same Bravais lattice. Notice that the discontinuities exhibited by some phonon branches in the $\mathrm{X}-\Gamma$ and $\Gamma$-Z directions when they intersect at the $\Gamma$-point are related to the LO-TO splitting of the optical infrared phonons.

mixture of partial bands: $\mathrm{H}, \mathrm{O}$ and $\mathrm{P}$ partial bands mix in the former, while $\mathrm{O}, \mathrm{K}$, and $\mathrm{P}$ partial bands are mixed in the latter. These bands are separated by frequency gaps. The largest gap appears between the HB and HS bands in the region (1400-2450) $\mathrm{cm}^{-1}$, while minor gaps arise in the regions $(1170-1200) \mathrm{cm}^{-1}$ and $(620-750) \mathrm{cm}^{-1}$ (see Fig. 2).

Our results for the total and partial phonon DOS in the FE phase of KDP are in good qualitative agreement with the corresponding ab initio results obtained using the $\mathrm{XC}$ functional PBE+D3 which includes empirical dispersion corrections ${ }^{91}$. Particularly, the bands and gaps distributions agree well in both calculations, with the only exception of the HS band that is shifted $\approx 500$ $\mathrm{cm}^{-1}$ towards smaller frequencies in the PBE+D3 approach, which should be attributable to the neglect of non-local vdW corrections. Moreover, our AI results for the bands and gaps distributions in the FE phase are also in good overall agreement with infrared ${ }^{18}$ and Raman ${ }^{83}$ experiments. In particular, our assignments for the frequency spectrum up to $\approx 1400 \mathrm{~cm}^{-1}$ agree remarkably well with the infrared spectrum at $80 \mathrm{~K}$ (with the polarization along the FE axis) reported in Fig. 3 of Ref. ${ }^{18}$. For instance, the low frequency structure up to $700 \mathrm{~cm}^{-1}$ in the imaginary part of the dielectric function detected by infrared experiments with the polarization along the FE axis consists of three peaks: the external one centered at $\approx 200 \mathrm{~cm}^{-1}$ and two IM peaks at $\approx 300$ and $500 \mathrm{~cm}^{-1}$ (see Fig. 3 of Ref. ${ }^{18}$ ). This is in good agreement with our AI finding of a low frequency "E+IMB" band with three peaks also: the external one at $\approx 150 \mathrm{~cm}^{-1}$, and the IMB band with peaks at $\approx 350$ and $500 \mathrm{~cm}^{-1}$. Moreover, as also shown in Fig. 3 of Ref. ${ }^{18}$, infrared measurements unambiguously detect three main resonant peaks in the region $(700-1400) \mathrm{cm}^{-1}$ : one at $\approx 900 \mathrm{~cm}^{-1}$ (IM phonon) and the other two at $\approx 1000$ and $1300 \mathrm{~cm}^{-1}$, which were assigned to $\mathrm{O}-\mathrm{H}$ bending modes. These measurements are in very good correspondence with our AI finding of a "IMS + HB" band with three main peaks at $\approx 850 \mathrm{~cm}^{-1}$ $(\mathrm{IMS}), \approx 1000 \mathrm{~cm}^{-1}(\mathrm{IMS}+\mathrm{HB})$, and $\approx 1300($ pure HB$)$ (see Fig. 2).

Notice that our band distribution up to $1400 \mathrm{~cm}^{-1}$ is also qualitatively similar to the distribution obtained in infrared measurements with the electric field polarized in the plane perpendicular to the FE axis (see Fig. 9 of Ref. ${ }^{18}$ ). Both infrared spectra (with polarizations parallel and perpendicular to the FE axis) show the existence of two gaps for frequencies lower than $1500 \mathrm{~cm}^{-1}$. One gap is located in the region $\approx 550-800 \mathrm{~cm}^{-1}$, and the other one is very small, with a width of scarcely $\approx 20$ 

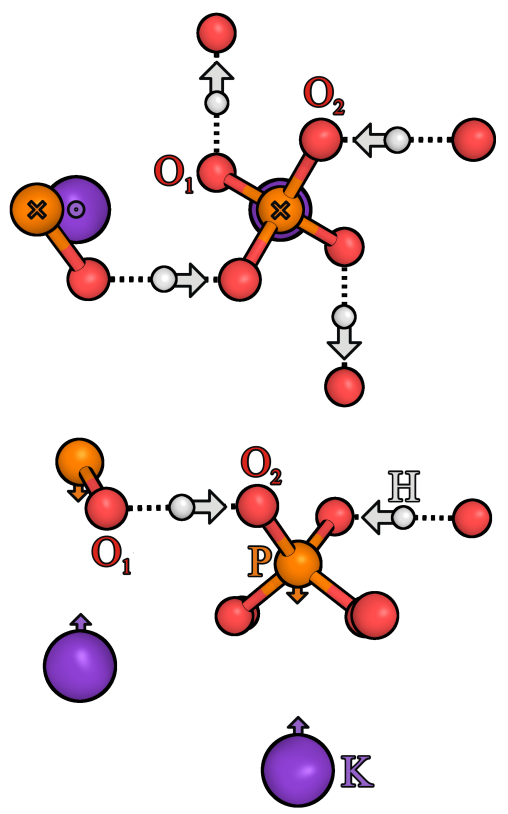

Figure 3: Schematic representation of the atomic displacements for the FE mode of KDP viewed along the FE $c$-axis (top panel) and perpendicular to it (bottom panel). The largest motion is that of the hydrogen atoms.

$\mathrm{cm}^{-1}$, and it is centered at $\approx 1200 \mathrm{~cm}^{-1} \cdot{ }^{18}$ Raman experiments also report a gap that extends from $\approx 600$ to $800 \mathrm{~cm}^{-1} .{ }^{83}$ Our calculated gaps in the regions (620-750) $\mathrm{cm}^{-1}$ and (1170-1200) $\mathrm{cm}^{-1}$ are in good agreement with those observed in infrared and Raman experiments.

Infrared measurements with the polarization perpendicular to the FE axis exhibit two shallow and strongly damped bands in the region $1500-2500 \mathrm{~cm}^{-1}$ with a small gap centered at $\approx 2000 \mathrm{~cm}^{-1}$. These bands were attributed to the H-stretching modes (see Fig. 9 of Ref. ${ }^{18}$ ). This contrasts with our AI result of a narrow HS bimodal band centered at $\approx 2600 \mathrm{~cm}^{-1}$, although the maximum frequencies obtained for the HS modes agree qualitatively with those observed experimentally. A discrepancy with experiment is also observed in the region where calculations predict a gap, although the calculated position of the gap center agrees with that reported in infrared experiments. The strong damping observed experimentally for these bands suggests the existence of strong anharmonic couplings with other modes. This observation could explain the disagreement between the experimental data and the present AI calculations, which do not take into account anharmonicity.

\section{Specific heat and Debye temperature}

Once the information of the phonon DOS is obtained from the AI calculations (see results in Fig. 2 for the FE phase), we are in a position to compute thermody-
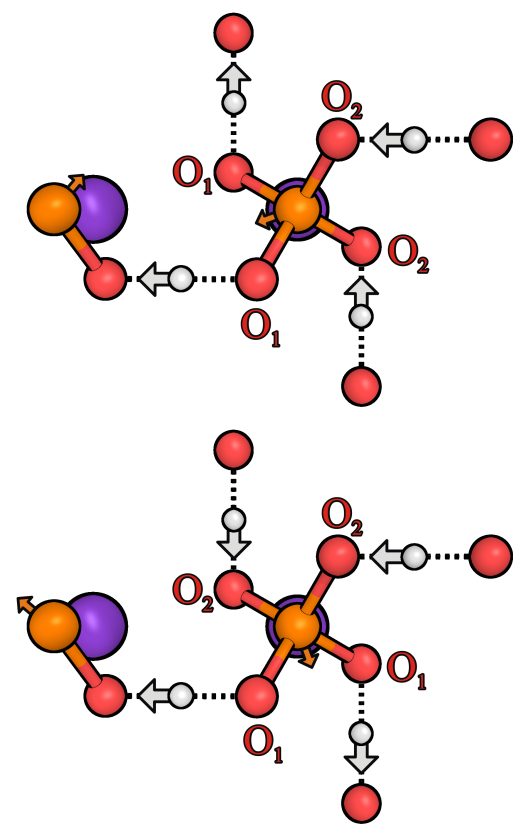

Figure 4: Schematic representations of the atomic displacements for the 2-fold degenerate unstable E-mode of the PE phase viewed along the FE $c$-axis. Both degenerate modes are shown in the top and bottom panels. The two $\mathrm{H}$ atoms approaching the $\mathrm{PO}_{4}$ group are one at its top and the other at its bottom. H atoms: white spheres, $\mathrm{P}$ atoms: orange spheres, and K atoms: violet spheres (see also the corresponding labels in Fig. 3).

namic properties that depend on the phonon spectrum. In this vein, we have evaluated the specific heat at constant volume as a function of temperature, $\mathrm{C}_{v}(\mathrm{~T})$, as an integral property using the AI total DOS expressed as a function of frequency $g(\omega) .{ }^{92-96}$ The results are plotted as a function of $\mathrm{T}$ in Fig. 5. Since in a solid at low temperature ${ }^{47,94} \mathrm{C}_{v} \approx \mathrm{C}_{p}$, our AI results of $\mathrm{C}_{v}(\mathrm{~T})$ can be compared directly with experimental data for $\mathrm{C}_{p}(\mathrm{~T})$. Hereafter, we take $\mathrm{C} \equiv \mathrm{C}_{v} \approx \mathrm{C}_{p}$.

As shown in Fig. 5, the $\mathrm{AI}$ results for $\mathrm{C}$ are in good overall agreement with the experimental data ${ }^{97-99}$ apart from a very small underestimation for temperatures above $\approx 40 \mathrm{~K}$. Notice that the experimental curve of Ref. ${ }^{97}$ exhibits a peak at $\approx 120 \mathrm{~K}$ that corresponds to the FE-PE phase transition, and which cannot be accounted for by the harmonic approximation used in this work. Moreover, since the present results for $\mathrm{C}$ were obtained using the total DOS in the FE phase (see Fig. 2), the theoretical curve shown in Fig. 5 has only a physical meaning for temperatures below $\mathrm{T}_{c} \approx 120 \mathrm{~K}$. The continuation of the curve by dotted lines for higher temperatures is only shown for completeness.

In order to monitor the Debye-like $\mathrm{C} \propto T^{3}$ behavior at low temperatures, we have also calculated the effective (or equivalent) Debye temperature $\Theta_{D}(T)$ which is associated to the value of the specific heat at each temperature. ${ }^{92,100}$ This magnitude and the values of 


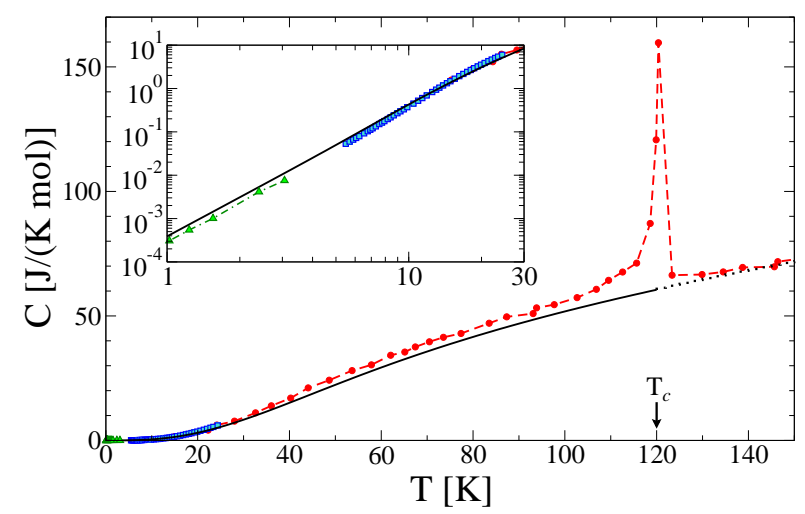

Figure 5: Ab initio results for the specific heat of KDP (solid line) in the FE phase. We also show the experimental results with red solid circles and dashed line (Ref. ${ }^{97}$ ), with blue open squares (Ref. ${ }^{98}$ ), and with green open triangles (Ref. ${ }^{99}$ ). Notice the peak in the experimental data of Ref. ${ }^{97}$ which corresponds to the $\mathrm{FE}-\mathrm{PE}$ phase transition at $\mathrm{T}_{c} \approx 120 \mathrm{~K}$. In dotted line is shown the continuation of the theoretical curve for temperatures higher than $\mathrm{T}_{c}$. In the inset, the abscissa displays the temperature in logarithmic scale from $1 \mathrm{~K}$ to 30 $\mathrm{K}$, while the ordinate shows the specific heat in logarithmic scale.

$\mathrm{C} / T^{3}$ are plotted as a function of $\mathrm{T}$ in Fig. 6, and compared to the corresponding values derived from the specific heat experimental data ${ }^{97-99}$.

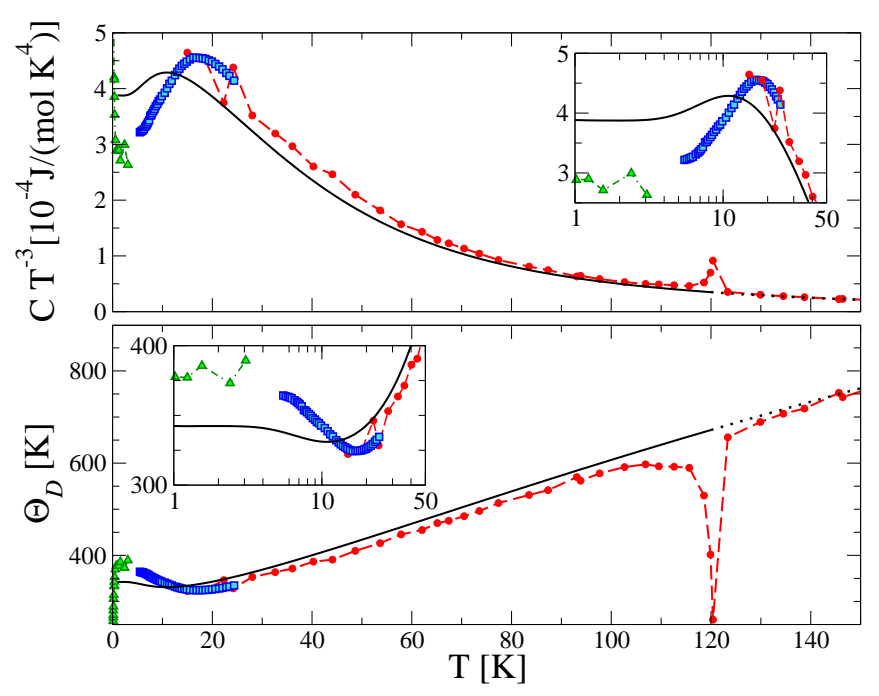

Figure 6: Top panel: $\mathrm{C} / T^{3}$ as a function of $\mathrm{T}$. Bottom panel: equivalent Debye temperature $\Theta_{D}(T)$ as a function of $\mathrm{T}$. The theoretical results for the AI calculations are shown in solid line. The experimental results are plotted with red solid circles and dashed line (Ref. ${ }^{97}$ ), with blue open squares (Ref. ${ }^{98}$ ), and with green open triangles (Ref. ${ }^{99}$ ). In the insets to both panels, the abscissa displays the temperature in logarithmic scale from $1 \mathrm{~K}$ to $50 \mathrm{~K}$.

We observe in Fig. 6 that the theoretical values of $\Theta_{D}(T)$ show a very small Debye-region of $\approx 4 \mathrm{~K}$ where $\Theta_{D}(T) \approx \Theta_{D}(T=0 \mathrm{~K})$ (independent of $\mathrm{T}$ ), in which case the Debye model is strictly valid (see the inset to the bottom panel in Fig. 6). The same constant region is clearly seen in the theoretical curve for $\mathrm{C} / T^{3}$, portrayed in the inset to the top panel of Fig. 6. We also observe a drop of $\approx 3 \%$ in the theoretical $\Theta_{D}(T)$ from the zero-temperature value $\left(\Theta_{D}(T=0 \mathrm{~K}) \approx 340\right.$ $\mathrm{K})$ to the minimum value at $\approx 12 \mathrm{~K}$ which is typical at low temperatures. ${ }^{92,100}$ These features can be ascribed to the onset of curvature in the acoustic dispersion curves at very low frequency produced by strong hybridizations with the low-frequency optical branches towards the Brillouin-zone boundary (see Fig. 2).

The theoretical $\Theta_{D}(T)$ and $\mathrm{C} / T^{3}$ values follow the same qualitative behavior with temperature as the corresponding experimental curves ${ }^{97-99}$ at temperatures higher than $1 \mathrm{~K}$ (see both panels of Fig. 6 and their insets). At lower temperatures, the experimental data of Ref. ${ }^{99}$ show an anomalous non-Debye behavior (see the sudden large drop in the experimental values for $\Theta_{D}(T)$ at very low temperatures in Fig. 6). This behavior corresponds to a linear term in $\mathrm{T}$ for the specific heat which is attributed to non-phononic dipolar defects present at very low temperatures, and which might lead to glassy behavior if in sufficient concentration (see discussion in Ref. $^{99}$ ).

A good agreement between the theoretical value $\Theta_{D}(T=0 \mathrm{~K}) \approx 340 \mathrm{~K}$ in the Debye region and the corresponding value reported in the experiment of Ref. ${ }^{99}$ $\left(\Theta_{D}^{\exp }(T=1 \mathrm{~K}) \approx 375 \mathrm{~K}\right)$, is observed in the inset to the bottom panel of Fig. 6. This agreement is also observed for the extent of the small Debye-region $(\approx 5 \mathrm{~K}$ in the experiment considering the results of Ref. ${ }^{98,99}$ and $\approx 4 \mathrm{~K}$ in the theory, as shown in the insets to Fig. 6). Moreover, the experimental value of $\Theta_{D}(T)$ reaches a minimum value at $\approx 16 \mathrm{~K}$ (see the experimental data of Ref. $^{98}$ in the inset to the bottom panel of Fig. 6), which corresponds well with the theoretical minimum located at $\approx 12 \mathrm{~K}$. The magnitude of the drop in $\Theta_{D}(T)$, however, is underestimated by the theory.

\section{The ferroelectric phonon in the PE phase and its anharmonic coupling to other modes}

The $\mathrm{B}_{2}$ FE soft-mode of the PE phase (see Fig. 3) couples with other modes when the atoms are moved with amplitudes beyond the region of validity of the harmonic approximation. Here, we extend the preliminary analysis of such coupling effects presented in Ref. ${ }^{32}$, which was of limited extent.

To study the couplings to the $\Gamma$-point modes we displaced the atoms according to the FE mode with increasing amplitude. We then allowed the atoms in the orthogonal subspace to relax while constraining the amplitude of the FE mode. Finally, the obtained displacement pattern $\mathbf{u}_{p}^{F E}$ (coupl) was projected onto the eigenvectors corresponding to the other $\Gamma$-point modes $j$. Here the index $p$ denotes the atoms of the primitive cell. Using the or- 
thogonality condition for the displacements, the projection is given by:

$$
P_{j}(\Gamma)=\sum_{p} m_{p} \mathbf{u}_{p}(\Gamma, j) \cdot \mathbf{u}_{p}^{F E}(\operatorname{coupl})
$$

In this way, the sum of the squares of the projections, $\left|P_{j}(\Gamma)\right|^{2}$, over all the $\Gamma$-point modes $j$, amounts to one. Fig. 7 shows precisely the square of the projection of the five modes that couple most significantly to the FE mode, as functions of the constrained FE-mode amplitudes. We would like to notice here, that the projections obtained are an indication of the relevance of the coupling between the FE mode and other modes, but not exactly a measure of the anharmonic coupling between them which could be obtained for example within the effective Hamiltonian $\operatorname{approach}^{101}$.

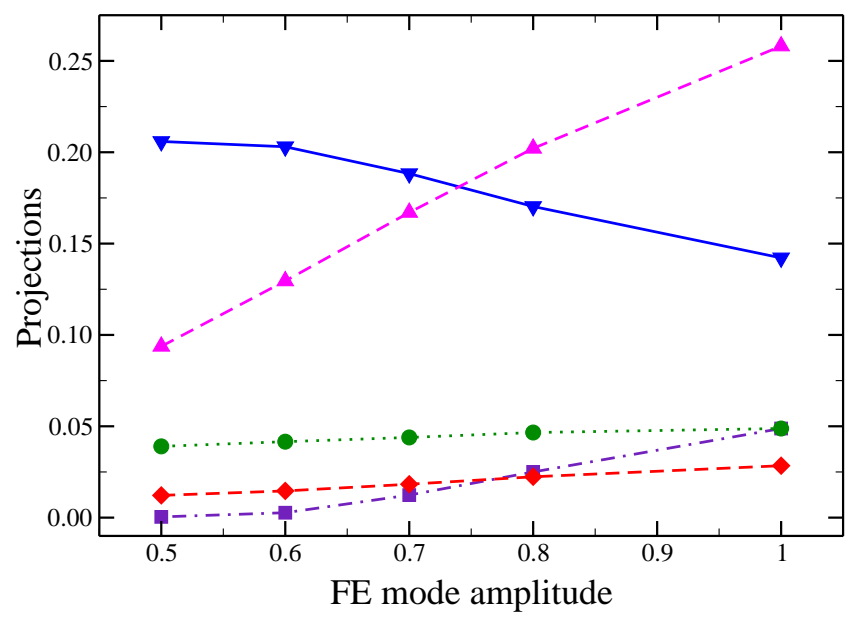

Figure 7: Couplings at various amplitudes of the FE mode with the five most strongly coupled $\Gamma$-modes. $B_{2}$ symmetry: $\mathrm{K}-\mathrm{PO}_{4}$ stretching at $189 \mathrm{~cm}^{-1}$ (blue inverted triangles and solid line), and $\mathrm{PO}_{4}$ distortion with $\mathrm{P}$ moving along $z$ at 533 $\mathrm{cm}^{-1}$ (green solid circles and dotted line). $\mathrm{A}_{1}$ symmetry: inphase $\mathrm{PO}_{4}$ distortion at $295 \mathrm{~cm}^{-1}$ (violet solid squares and dotted-dashed line), out-of-phase $\mathrm{PO}_{4}$ librations around the $c$-axis at $543 \mathrm{~cm}^{-1}$ (magenta solid triangles and dashed line), and in-phase $\mathrm{PO}_{4}$ breathing at $917 \mathrm{~cm}^{-1}$ (red solid diamonds and dashed line). In-phase and out-of-phase refer to the two adjacent $\mathrm{PO}_{4}$ units in the unit cell. The horizontal axis is scaled so that the unit value corresponds to the displacement of the FE mode at the minimum-energy configuration.

One of the two most strongly coupled modes, the $\mathrm{B}_{2}$ one at $189 \mathrm{~cm}^{-1}$ (blue inverted triangles in Fig. 7), belongs to the same representation of the FE mode, and corresponds to a $\mathrm{K}^{+}-\mathrm{H}_{2} \mathrm{PO}_{4}^{-}$optical stretching mode along the $c$-axis (see top of Figure 8 and the ET mode of $\mathrm{B}_{2}$ symmetry reported in Table $\mathrm{V}$ ). This motion also involves $\mathrm{H}$ displacements with projections along the $\mathrm{O}-\mathrm{H}-\mathrm{O}$ bonds displaying the same pattern as in the FE mode. The projection of the FE mode onto this mode decreases with increasing amplitude, at the expenses of the other modes.

Quite the contrary occurs with the other strongly coupled vibration, an $\mathrm{A}_{1}$ mode at $543 \mathrm{~cm}^{-1}$ (magenta solid triangles in Fig. 7, see also Table V), whose participation in the projection increases linearly with the amplitude of the FE mode displacement. This mode involves librations of neighboring $\mathrm{H}$-bonded $\mathrm{PO}_{4}$ groups in opposite direction, accompanied by a bending of the O-H-O bridges (see top panel in Fig. 9). This mode is often described as a rotation of the tetrahedra. It is well-known that, in hydrogen bonds, $\mathrm{O}-\mathrm{O}$ and $\mathrm{O}-\mathrm{H}$ distances are intimately related. As the proton moves away from the center of the bond, the $\mathrm{O}-\mathrm{O}$ distance should increase, and one way of achieving this is by rotating the tetrahedra in opposite directions. Hence, it is not surprising that these two modes are strongly coupled, and increasingly more for larger amplitudes.

Less strongly coupled to the FE mode is another $\mathrm{B}_{2}$ one at $533 \mathrm{~cm}^{-1}$ (green solid circles in Fig. 7), which involves a large quadrupole distortion of the $\mathrm{PO}_{4}$ tetrahedra $\left(\nu_{4}\right.$ IMB mode, see Table $\mathrm{V}$ ), with the $\mathrm{P}$ moving along the $c$-axis and the oxygens nearly perpendicular to it. The $\mathrm{H}$-displacement pattern is similar to that of the FE mode (see bottom panel in Figure 8).

Rather weakly coupled to the FE mode are two other internal $\mathrm{A}_{1}$ modes, at $295 \mathrm{~cm}^{-1}$ and $917 \mathrm{~cm}^{-1}$ (violet solid squares and red solid diamonds, respectively, in Fig. 7). The first one involves librations of the $\mathrm{PO}_{4}$ tetrahedra similar to the strongly coupled $\mathrm{A}_{1}$ mode at $543 \mathrm{~cm}^{-1}$, but with very little stretching of the $\mathrm{O}-\mathrm{H}-\mathrm{O}$ bridges. The mode at $917 \mathrm{~cm}^{-1}$ has a similar pattern (shown at the bottom of Fig. 9), but now the $\mathrm{PO}_{4}$ breathing and the stretching of the $\mathrm{O}-\mathrm{H}-\mathrm{O}$ bridges dominate over the librations.

It has been suggested that the coupling of the FE softmode with the phonons depicted in Fig. 8 should play an important role in the mechanism of the ferroelectric phase transition in KDP. ${ }^{18,102,103}$ Katiyar et al. ${ }^{102}$ were the first to interpret their Raman spectra for KDPisomorphic arsenate crystals in terms of the coupling between a heavily overdamped, strongly temperature dependent $\mathrm{B}_{2}$ mode (denoted as the tunneling mode and assigned to the FE mode in KDP), and another lowfrequency underdamped $\mathrm{B}_{2}$ mode. The same approach applied to the Raman results for $\mathrm{KDP}^{24,103}$ indicates that the $\mathrm{FE}$ mode is coupled to a $\mathrm{B}_{2}$ mode in the $\mathrm{PE}$ phase at a nearly temperature-independent frequency of $\approx 180$ $\mathrm{cm}^{-1}$. This is in excellent agreement with our finding of a strong coupling with the $\mathrm{B}_{2}$ mode at $189 \mathrm{~cm}^{-1}$. This is also supported by strong indications of a high anharmonicity associated to the optical $\mathrm{B}_{2}$ phonon at $\approx 180$ $\mathrm{cm}^{-1}$, which is manifested in a broad signal in the Raman spectra (the full linewidth at half-maximum is found to be $\left.\approx 27 \mathrm{~cm}^{-1}\right) .{ }^{24}$ Notice also that the corresponding ET $\mathrm{A}_{1}$ mode found at $\approx 209 \mathrm{~cm}^{-1}$ in the infrared measurements at very low $\mathrm{T}$ in the $\mathrm{FE}$ phase (our calculated value for this mode is $185 \mathrm{~cm}^{-1}$, see Table V) displays 


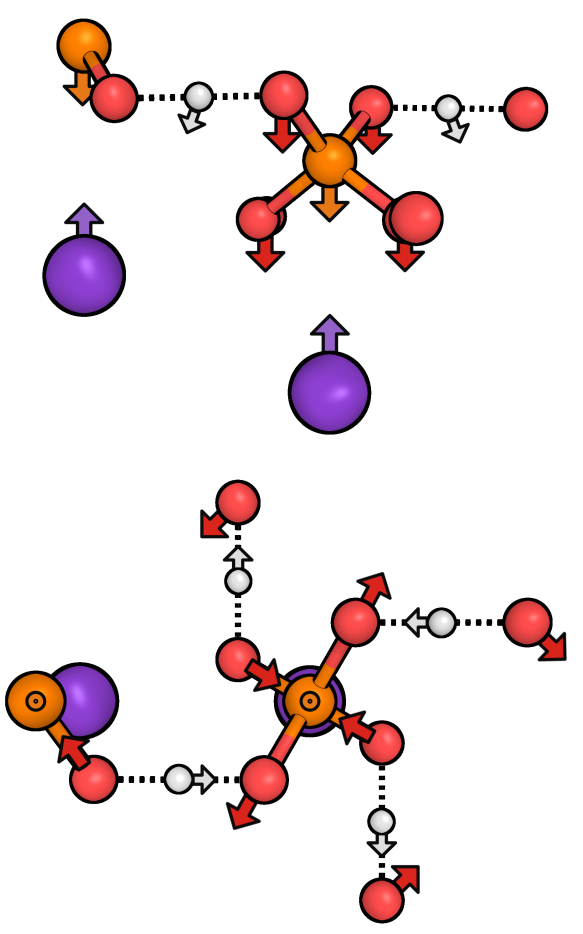

Figure 8: $\mathrm{B}_{2}$ modes in the $\mathrm{PE}$ phase that couple significantly to the FE soft-mode. Top panel: schematic side view (perpendicular to the $c$-axis) of the ET $z$-stretching mode at 189 $\mathrm{cm}^{-1}$. Bottom panel: schematic view along the $\mathrm{FE} c$-axis of the $\nu_{4}$ IMB mode at $533 \mathrm{~cm}^{-1}$, where only the $\mathrm{P}$ atom moves significantly along $z$. H atoms: white spheres, $\mathrm{O}$ atoms: red spheres, $\mathrm{P}$ atoms: orange spheres, and $\mathrm{K}$ atoms: violet spheres (see also the corresponding labels in Fig. 3).

a pronounced softening as the temperature increases approaching the phase transition, which is also indicative of a strong anharmonic behavior. ${ }^{18}$

Moreover, the coupling of the FE soft-mode with the quadrupolar, internal $\left(\nu_{4}\right) \mathrm{B}_{2}$ mode at $533 \mathrm{~cm}^{-1}$ shown at the bottom of Figure 8 (green solid circles in Fig. 7) has been suggested by infrared measurements. ${ }^{18,88}$ For instance, the infrared experiments of Ref. ${ }^{18}$ show a softening of the $\nu_{4}$ mode in the FE phase as the temperature approaches $T_{c}$. This is in accordance with the important increase of the oscillator strength for this mode as the phase transition is reached. Notice that the $\nu_{4}$ phonon detected by the infrared measurements at $\approx 440 \mathrm{~cm}^{-1}$ at low temperature in the $\mathrm{FE}$ phase corresponds in fact to a phonon of $\mathrm{A}_{1}$ symmetry, which transforms to the quadrupolar $\left(\nu_{4}\right) \mathrm{B}_{2}$ mode in the $\mathrm{PE}$ phase (see Table $\mathrm{V})$. Therefore, our results of strong couplings between the FE soft-mode and the ET and $\nu_{4} \mathrm{~B}_{2}$-phonons are fully consistent with the experimental infrared data that shows strong couplings between these phonons. ${ }^{18,88}$

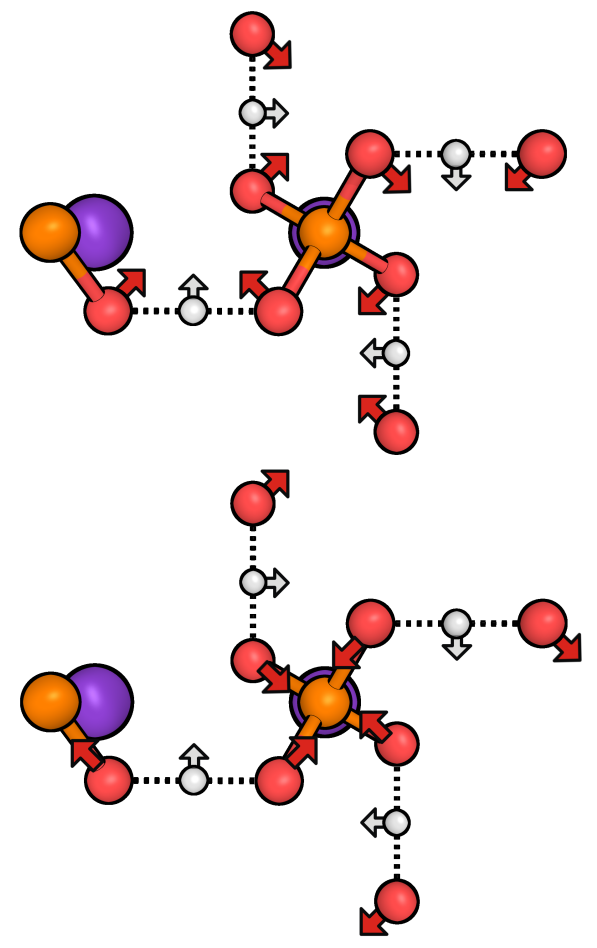

Figure 9: Schematic top views of the atomic displacements for the $A_{1}$ optical modes of the PE phase that couple significantly to the FE one: the strong coupled IMB mode at $543 \mathrm{~cm}^{-1}$ (top panel) and the weak coupled mode at $917 \mathrm{~cm}^{-1}$ (bottom panel). $\mathrm{H}$ atoms: white spheres, $\mathrm{O}$ atoms: red spheres, $\mathrm{P}$ atoms: orange spheres, and $\mathrm{K}$ atoms: violet spheres (see also the corresponding labels in Fig. 3).

\section{SUMMARY}

In this paper we have presented a comprehensive study of the structural and vibrational properties of KDP, as an emblematic example of a large class of hydrogen-bonded ferroelectric materials. Experimental data has been available for many decades, and has been interpreted, in particular the large isotope effect in the ferroelectric transition temperature $\mathrm{T}_{c}$, in terms of a variety of phenomenological models. Early classical models proposed in the forties by Slater and Takagi ${ }^{85,90}$ were extended in the sixties to the quantum-mechanical (tunnelling) domain by Blinc, Svetina, and De Gennes ${ }^{7,17,104}$. These models lacked an important ingredient that was identified only in the late eighties by Nelmes et al. through very sensitive neutron scattering experiments: ${ }^{13}$ isotope effects were accompanied by significant geometric rearrangements in the hydrogen-bond and phosphate geometries. These observations led to another class of models that incorporate geometric effects, such as the strong dipole-proton coupling model ${ }^{105}$ and variants ${ }^{89}$. These new models succeeded in harmonizing a substantial set of experimental results, and shed light into the matter of the character of the FE phase transition, whether orderdisorder or displacive. They did, however, remain at the 
phenomenological level, with parameters derived from experimental data.

In the past decades, ab initio calculations ${ }^{3,15,31}$ contributed to put these models on a firmer ground, by generating model- and experiment-independent data and parameters. However, these calculations based on semilocal approximations to DFT proved challenging when it came to comparing with experimental data, in particular internal geometries. There are two main reasons for this, one is that the choice of the exchange-correlation functional is not immaterial to the geometry, as has been recently shown in calculations for the related compound $\mathrm{CDP}^{38}$. The second one is that electronic structure calculations do not include quantum nuclear (isotope) effects, which we know are important from experimental data obtained for KDP and DKDP.

In this work we have addressed these two issues by computing the internal geometry and globaldisplacement energy barriers of KDP for a wide range of density functionals, including standard GGA, GGA supplemented with both empirical and ab initio van der Waals corrections, and hybrid DFT-Hartree Fock functionals. In addition we have computed the geometry and barrier also at the MP2 quantum chemical level. Barriers range from 25 to $250 \mathrm{meV}$, while differences in geometric parameters like the $\mathrm{O}-\mathrm{O}$ distance, can vary by up to 0.12 $\AA$ in the FE phase.

These ranges encompass the experimental values, but selecting a functional based solely on this is misleading, as these calculations do not take into account quantum nuclear delocalization. In the case of hydrogen bonds, nuclear quantum effects are known to shorten the bonds, which in turn modify the phonon spectrum. ${ }^{39}$ Computing the geometries at the first-principles level, selfconsistently in the presence of nuclear quantum effects is computationally very demanding, especially for hybrid functionals. This could be done using a nuclear wave function approach such as that in Ref. ${ }^{39}$ or Ref. ${ }^{106}$, or ab initio path integral molecular dynamics ${ }^{107}$ or Monte Carlo $^{79}$ (PIMC) approaches. In the present work we opted for a PIMC strategy but on a three-site model representing the $\mathrm{O}-\mathrm{H} \cdots \mathrm{O}$ hydrogen bond, adjusted to the geometries and barriers determined without quantum corrections. Through this procedure we corrected a posteriori the internal geometries of KDP and DKDP, as shown in Table III, and selected the functional that provided the best agreement for the $\mathrm{FE}$ and $\mathrm{PE}$ phase of both, KDP and DKDP, which turned out to be the non-local van der Waals functional vdW-DF. ${ }^{58,59}$

With this choice of functional, we computed the phonon modes at the $\Gamma$-point of the Brillouin zone for all irreducibles representations in the $\mathrm{PE}$ and $\mathrm{FE}$ phase, and compared them to the frequencies measured in infrared and Raman experiments. ${ }^{18,24-26,83}$ The agreement is remarkably good, thus allowing us to assign the various features of the vibrational spectra. In the PE phase we identified three unstable modes, one corresponding to the FE mode and two degenerate ones in the $x y$-plane that are associated to the lateral Slater configurations, and produce an in-plane polarization. The latter were linked to a low-frequency strongly-damped feature in the infrared spectrum. ${ }^{29,88}$ These three modes together with a highfrequency tetrahedron breathing mode can be associated with the four basic configurations in the Slater model, while non- $\Gamma$ phonons would enhance this model with the Takagi configurations in which one of three atoms approach the phosphate. A group of bending modes of the hydrogen bonds (a dispersion-less band in the full phonon dispersions of Fig. 2) lies, in the FE phase, about $1000 \mathrm{~cm}^{-1}$ below and well-separated from the stretching modes. These modes are preceded by two groups (or bands) of internal molecular phonons in the regions 300$600 \mathrm{~cm}^{-1}$ and $750-1200 \mathrm{~cm}^{-1}$, that involve deformations of the $\mathrm{PO}_{4}$ tetrahedra. Finally, modes below $300 \mathrm{~cm}^{-1}$ involve the motion of the $\mathrm{K}^{+}$ions.

Phonon dispersions allowed us to compute the phonon density of states, which was then used to obtain the specific heat and the Debye temperature in the FE phase and to compare with experimental data. ${ }^{97}$. The agreement is again remarkably good, including a small region at low temperatures where $\mathrm{C} \propto T^{3}$ and the Debye model is strictly valid. The peak at $120 \mathrm{~K}$ is associated to the FE-PE transition and is not taken into account in the calculations.

Finally, we studied the coupling between the FE mode and other modes at the $\Gamma$-point by constraining the amplitude of the FE mode and projecting the displacement pattern onto the eigenvectors. This analysis showed that there are two modes that couple significantly: the first one belongs to the same representation and can be characterised as an optical stretching of the $\mathrm{K}^{+}-\mathrm{PO}_{4}^{-}$units along the $c$-axis. This coupling is responsible for the development of polarization perpendicular to the $x y$-planes that contain the H-bonds, and is related to a large offdiagonal Born effective charge on the hydrogen atoms, $Z_{H, x z}^{*}$ that was reported in Ref. ${ }^{32}$ The second stronglycoupled mode is an out-of-phase libration (rotation) of neighboring $\mathrm{PO}_{4}$ tetrahedra. The rotation of the tetrahedra in opposite directions modulates the O-O distance, which is intimately connected to the geometry of the hydrogen bond; the smaller $d_{O O}$ the smaller $\delta$, and the stronger the bond. The participation of these two modes follows opposite trends with amplitude, i.e. with increasing temperature; the polarization mode couples less, while the rotation of the tetrahedra becomes more important. Therefore, any model aimed at describing the isotope effect will have to take into account at least three fully-coupled modes.

\section{Acknowledgments}

R.M., F.T., J.L. and S.K. acknowledge support from Consejo Nacional de Investigaciones Científicas y Técnicas (CONICET), Argentina. 
1 M. E. Lines and A. M. Glass, Principles and Applications of Ferroelectric and Related Materials, (Clarendon, Oxford, 1977).

2 G. Busch and P. Scherrer, Naturwiss. 23, 737 (1935).

3 S. Koval, J. Kohanoff, J. Lasave, G. Colizzi, R. L. Migoni, Phys. Rev. B 71, 184102 (2005).

${ }^{4}$ R. Blinc, Advanced Ferroelectricity, (Oxford University Press, New York, 2011).

5 M.I. McMahon et al., Nature (London) 348, 317 (1990).

${ }^{6}$ R. J. Nelmes, M. I. McMahon, R. O. Piltz, and N. G. Wright, Ferroelectrics 124, 355 (1991).

7 R. Blinc, J. Phys. Chem. Solids 13, 204 (1960).

8 K. Kobayashi, J. Phys. Soc. Jpn. 24, 497 (1968).

9 M. Tokunaga and T. Matsubara, Ferroelectrics 72, 175 (1987).

10 R. Blinc and B. Zeks, Ferroelectrics 72, 193 (1987).

11 M. Ichikawa, K. Motida and N. Yamada, Phys. Rev. B 36, 874 (1987)

12 Z. Tun et al., J. Phys. C: Solid State Phys. 21, 245 (1988).

13 R.J. Nelmes, J. Phys. C: Solid State Phys. 21, L881 (1988).

14 G.F. Reiter, J. Mayers, and P. Platzman, Phys. Rev. Lett. 89, 135505 (2002).

15 S. Koval, J. Kohanoff, R. L. Migoni and E. Tosatti, Phys. Rev. Lett. 89, 187602 (2002).

16 M. Tokunaga and I. Tatsuzaki, Phase Transitions 4, 97 (1984).

17 P. G. De Gennes, Solid State Commun. 1, 132 (1963).

18 P. Simon, F. Gervais, and E. Courtens, Phys. Rev. B 37, 1969 (1988).

19 J. J. Kweon, R. Fu, E. S. Choi, and N. S. Dalal, J. Phys.: Condens. Matter 29, 16LT01 (2017).

20 A. A. Volkov, G. V. Kozlov, S. P. Lebedev and, A. M. Prokhorov, Ferroelectrics, 25, 531 (1980).

21 M. Kasahara and I. Tatsuzaki, J. Phys. Soc. Jpn. 50, 3972 (1981).

${ }^{22}$ Y. Tominaga, H. Urabe, and M. Tokunaga, Solid State Commun. 48, 265 (1983).

${ }^{23}$ R. Blinc and B. Zeks, J. Phys. C 15, 4661 (1982).

${ }^{24}$ C. Y. She, T. W. Broberg, and D. F. Edwards, Phys. Rev. B 4, 1580 (1971).

25 Y. Tominaga, M. Kasahara, H. Urabe, and I. Tatsuzaki, Solid State Commun. 47, 835 (1983).

${ }^{26}$ K. C. Serra, F. E. A. Melo, J. Mendes Filho, F. A. Germano, and J. E. Moreira, Solid State Commun. 66, 575 (1988).

27 S. Shin, A. Sugawara, Y. Tezuka, and M. Ishigame, Solid State Commun. 71, 685 (1989).

28 B. A. Liu, H. L. Zhou, Q. H. Zhang, M. X. Xu, S. H. Ji, L. L. Zhu, L. S. Zhang, F. F. Liu, X. Sun, and X. G. Xu, Chin. Phys. Lett. 30, 067804 (2013).

29 B. Wyncke and F. Bréhat, J. Phys. C: Solid State Phys. 19, 2649 (1986).

30 T. Fujiwara, J. Phys. Soc. Japan 29, 1282 (1970).

31 J. Lasave, J. Kohanoff, R. L. Migoni, and S. Koval, Physica B 404, 2736 (2009).

32 G. Colizzi, J. Kohanoff, J. Lasave, and R. L. Migoni, Ferroelectrics 401, 200 (2010).

33 Ya. Shchur, Phys. Rev. B 74, 054301 (2006).

${ }^{34}$ Ya. Shchur, T. Bryk, I. Klevets, and A.V. Kityk, Comput. Mater. Sci. 111, 301 (2016).
35 B. Van Troeye, M. J. van Setten, M. Giantomassi, M. Torrent, G.M. Rignanese, and X. Gonze, Phys. Rev. B 95, $024112(2017)$

36 K. Lee, B. Kolb, T. Thonhauser, D. Vanderbilt, and D. C. Langreth, Phys. Rev. B 86, 104102 (2012).

37 K. T. Wikfeldt and A. Michaelides, J. Chem. Phys. 140, 041103 (2014).

38 J. Lasave, P. Abufager, and S. Koval, Phys. Rev. B 93, 134112 (2016).

39 I. Scivetti, N. Gidopoulos, and J. Kohanoff, Phys. Rev. B 78, 224108 (2008)

40 S. Baroni, S. de Gironcoli, A. dal Corso and P. Gianozzi, Rev. Mod. Phys. 73, 515 (2001).

${ }^{41}$ G. Kresse and J. Furthmuller, Comput. Mater. Sci. 6, 15 (1996).

42 G. Kresse and J. Furthmuller, Phys. Rev. B 54, 11169 (1996).

43 J. Hutter, M.Iannuzzi, F. Schiffmann, and J. VandeVondele, WIREs Comput Mol Sci 4, 15 (2014).

44 J. VandeVondele, M. Krack, F. Mohamed, M. Parrinello, T. Chassaing, and J. Hutter, Comput. Phys. Commun. 167, 103 (2005).

45 G. Lippert, J. Hutter, and M. Parrinello, Mol. Phys. 92, 477 (1997).

46 G. Lippert, J. Hutter, and M. Parrinello, Theor. Chem. Acc. 103, 124 (1999).

47 A. Togo and I. Tanaka, Scr. Mater. 108, 1 (2015).

48 G. Kresse, J. Furthmüller, J. Hafner, Europhys. Lett. 32, 729 (1995).

49 N. Troullier and J. L. Martins, Phys. Rev. B 43, 1993 (1991).

50 S. Goedecker, M. Teter, and J. Hutter, Phys. Rev. B 54, 1703 (1996).

51 C. Hartwigsen, S. Goedecker, and J. Hutter, Phys. Rev. B 58, 3641 (1998).

${ }^{52}$ M. Guidon, J. Hutter, and J. VandeVondele, J. Chem. Theory Comput. 6, 2348 (2010).

53 M. Guidon, J. Hutter, and J. VandeVondele, J. Chem. Theory Comput. 5, 3010 (2009).

54 V. Rybkin and J. VandeVondele, J. Chem. Theory Comput. 12, 2214 (2016).

55 M. del Ben, J. Hutter, and J. VandeVondele, J. Chem. Phys. 143, 102803 (2015).

${ }^{56}$ M. del Ben, J. Hutter, J. VandeVondele, J. Chem. Theory Comput. 9, 2654 (2013).

57 R. J. Nelmes, Z. Tun, and W. F. Kuhs, Ferroelectrics 71, 125 (1987).

58 M. Dion, H. Rydberg, E. Schroder, D. C. Langreth, and B. I. Lundqvist, Phys.Rev.Lett. 92, 246401 (2004).

59 J. Klimeš, D. R. Bowler, and A. Michaelides, Phys. Rev. B 83, 195131 (2011).

60 G. Román-Pérez and J. M. Soler, Phys. Rev. Lett. 103, 096102 (2009).

${ }^{61}$ K. Lee, E. D. Murray, L. Kong, B. I. Lundqvist, and D. C. Langreth, Phys. Rev. B 82, 081101 (2010).

62 J. Klimeš, D. R. Bowler, and A. Michaelides, J. Phys. Condens. Matter 22, 022201 (2010).

63 S. Grimme, J. Comp. Chem. 27, 1787 (2006).

64 A. Tkatchenko and M. Scheffler, Phys. Rev. Lett. 102, 073005 (2009).

65 J. P. Perdew, K. Burke, and M. Ernzerhof, Phys. Rev. 
Lett. 77, 3865 (1996).

${ }^{66}$ C. Adamo and V. Barone, J. Chem. Phys. 110, 6158 (1999).

67 J. Heyd, G. E. Scuseria, and M. Ernzerhof, J. Chem. Phys. 118, 8207 (2003).

68 J. Heyd, G. E. Scuseria, and M. Ernzerhof, J. Chem. Phys. 124, 219906 (2006).

69 Y. Zhao and D.G. Truhlar (2006), Theor. Chem. Acc. 120, 215 (2008).

70 J. Harl, L.. Schimka, and G. Kresse, Phys. Rev. B 81, 115126 (2010).

71 J. Lasave, S. Koval, N. S. Dalal, and R. L. Migoni, Phys. Rev. Lett. 98, 267601 (2007).

72 J. Lasave, S. Koval, R. L. Migoni, and N. S. Dalal, J. Chem. Phys. 135, 084504 (2011).

73 J.M. Robertson and A.R. Ubbelohde, Proc. R. Soc. London A 170, 222 (1939).

74 E. Matsushita and T. Matsubara, Prog. Theor. Phys. 67, 1 (1982); T. Matsubara and E. Matsushita, Prog. Theor. Phys. 71, 209 (1984).

75 O. Yanovitskii, G. Vlastou-Tsinganos, and N. Flytzanis, Phys. Rev. B 48, 12645 (1993).

76 Satoshi Tanaka, Phys. Rev. B 42, 10488 (1990).

77 C. Chakravarty, Int. Rev. Phys. Chem. 16, 421 (1997).

78 M. Takahashi and M. Imada, J. Phys. Soc. Jpn. 53, 3765 (1984).

79 R. O. Weht, J. Kohanoff, D. A. Estrín, and C. Chakravarty, J. Chem. Phys., 108, 8848 (1998).

${ }^{80}$ K. Röttger, A. Endriss, J. Ihringer, S. Doyle, and W. F. Kuhs, Acta Crystallogr. Sect. B 50, 644 (1994).

81 B. Pamuk, J. M. Soler, R. Ramírez, C. P. Herrero, P. W. Stephens, P. B. Allen, and M.-V. Fernández-Serra, Phys. Rev. Lett. 108, 193003 (2012)

82 M. S. Shur, Sov. Phys. Sol. State 8, 43 (1966); ibid 8, 1008 (1966); Sov. Phys. Cryst. 11, 394 (1966); ibid 12, 181 (1967)

83 J. P. Coignac and H. Poulet, J. Phys. (Paris) 32, 679 (1971).

84 W. Setyawan and S. Curtarolo, Comput. Mater. Sci. 49, 299 (2010).

85 J. C. Slater, J. Chem. Phys. 9, 16 (1941).

86 J. Lasave, S. Koval, N. S. Dalal, and R. L. Migoni, Phys. Rev. B 72, 104104 (2005) .
87 W. Reese, Phys. Rev. 181, 905 (1969); C. W. Fairall and W. Reese, Phys. Rev. B 11, 2066 (1975).

88 F. Bréhat and B. Wyncke, J. Phys. C: Solid State Phys. 21, 4853 (1988).

89 D. Merunka and B. Rakvin, Phys. Rev. B 66, 174101 (2002).

90 Y. Takagi, J. Phys. Soc. Japan 3, 271 (1948).

91 Y. Finkelstein, R. Moreh, S. L. Shang, Ya. Shchur, Y. Wang, and Z. K. Liu, J. Chem. Phys. 144, 054302 (2016).

92 P. Brüesch, Phonons: Theory and Experiments I, (Springer-Verlag, Berlin, 1982).

93 M. T. Dove, Introduction to Lattice Dynamics, (Cambridge University Press, Cambridge, 1993).

94 A. Togo, L. Chaput, I. Tanaka, and G. Hug, Phys. Rev. B 81, 174301 (2010)

95 H. Giefers, S. Koval, G. Wortmann, W. Sturhahn, E. E. Alp, and M. Y. Hu, Phys. Rev. B 74, 094303 (2006).

96 J. Lasave, F. Dominguez, S. Koval, M. G. Stachiotti and R. L. Migoni, J. Phys.: Condens. Matter 17, 7133 (2005).

97 C. C. Stephenson and J. G. Hooley, J. Am. Chem. Soc. 66, 1397 (1944).

98 W. N. Lawless and T. D. Lawless, Ferroelectrics 45, 149 (1982).

99 M. C. Foote and A. C. Anderson, Ferroelectrics 62, 11 (1985).

100 S. Koval, R. Burriel, M. G. Stachiotti, M. Castro, R. L. Migoni, M. S. Moreno, A. Varela, and C. O. Rodriguez, Phys. Rev. B 60, 14496 (1999).

101 K. M. Rabe and U. V. Waghmare, Ferroelectrics 151, 59 (1994).

102 R. S. Katiyar, J. F. Ryan and J. F. Scott, Phys. Rev. B 4, 2635 (1971).

103 C. Y. She, T. W. Broberg, L. S. Wall, and D. F. Edwards, Phys. Rev. B 6, 1847 (1972)

104 R. Blinc and S. Svetina, Phys. Rev. 147, 430 (1966).

105 H. Sugimoto and S. Ikeda, Phys. Rev. Lett. 67, 1306 (1991).

106 B. Monserrat, N. D. Drummond, and R. J. Needs, Phys. Rev. B 87, 144302 (2013).

107 D. Marx and M. Parrinello, J. Chem. Phys. 104, 4077 (1996). 